LA-UR-98-

January 1998

\title{
TASK COMPLETION REPORT FOR INVESTIGATING WHY OUTPUT SIGNAL-VARIABLE VALUES DIFFER FROM THEIR OUTPUT COMPONENT-PARAMETER VALUES IN TEST
}

Robert G. Steinke, TSA-10

\section{RECEIVED \\ n. 280.0 \\ 0871}

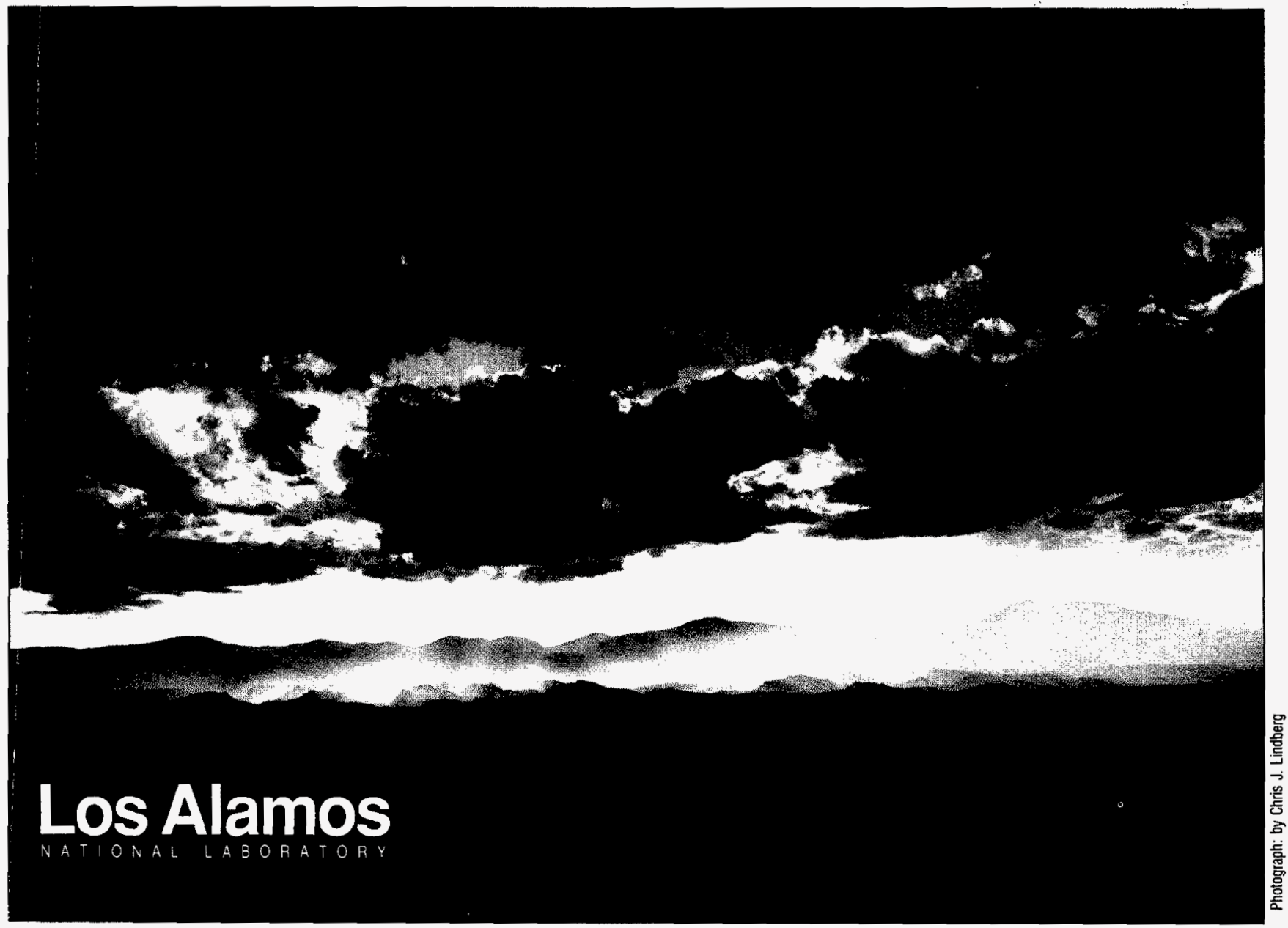

Los Alamos National Laboratory, an affirmative action/equal opportunity employer, is operated by the University of California for the U.S. Department of Energy under contract published form of this contribution, or to allow others to do so, for U.S. Government U.S. Government retains a nonexclusive, royalty-free license to publish or reproduce the as work performed under the auspices of the U.S. Department of Energy. Los Alamos Narional Laboratory strongly supports academic freedom and a researcher's right to publish; therefore, the Laboratory as an institution does not endorse the viewpoint of a publication or guarantee its technical correctness. 


$$
\text { LA- UR }--98-99
$$

TASK COMPLETION REPORT

FOR INVESTIGATING WHY OUTPUT SIGNAL-VARIABLE VALUES DIFFER FROM THEIR OUTPUT COMPONENT-PARAMETER

VALUES IN TEST PROBLEM MST2

by

Robert G. Steinke

September 10, 1997

\section{MASTER}

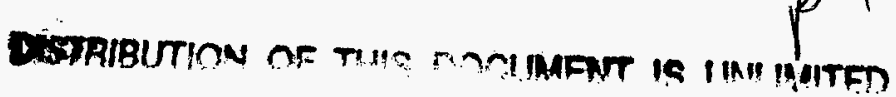




\section{DISCLAIMER}

This report was prepared as an account of work sponsored by an agency of the United States Government. Neither the United States Government nor any agency thereof, nor any of their employees, makes any warranty, express or implied, or assumes any legal liability or responsibility for the accuracy, completeness, or usefulness of any information, apparatus, product, or process disclosed, or represents that its use would not infringe privately owned rights. Reference herein to any specific commercial product, process, or service by trade name, trademark, manufacturer, of otherwise does not necessarily constitute or imply its endorsement, recommendation, or favoring by the United States Government or any agency thereof. The views and opinions of authors expressed herein do not necessarily state or reflect those of the United States Government or any agency thereof. 


\title{
TASK COMPLETION REPORT \\ FOR INVESTIGATING WHY OUTPUT SIGNAL-VARIABLE VALUES DIFFER FROM THEIR OUTPUT COMPONENT-PARAMETER VALUES IN TEST PROBLEM MST2
}

\author{
Robert G. Steinke
}

\begin{abstract}
Signal-variable values and their component-parameter values differ in an end-of-timestep edit to the TRCOUT and TRCGRF files because signal variables have beginning-of-timestep values, and component parameters have end-of-timestep values. Oscillatory divergence in the MST2 standard test problem after $9000 \mathrm{~s}$ occurs because of TRAC-P's numerical evaluation at a 1000 material Courant number. The magnitude of that divergence has diminished by a factor of 3.5 from Version 5.3.01 to 5.4.15 and by a factor of 25 from Version 5.4.15 to 5.4.28. That divergence can be eliminated by evaluating MST2 with a maximum material Courant number of 500 .
\end{abstract}

\subsection{INTRODUCTION}

Knolls Atomic Power Laboratory (KAPL) reported for standard test problem MST2 that TRAC-P Version 5.4.15 signal-variable values differ from their componentdata values output to the TRCOUT and TRCGRF files. The signal variables that were investigated have their additional signal-variable input data for file MST2 shown in Table I. On graphics parameter-vs-time plots, the signal-variable values lead their component-data values initially. At end-of-problem times 9300 to 10,000 $\mathrm{s}$, the plotted signal-variable values were shown to diverge from their componentdata values for the gas volume fraction, phasic and mixture mass flows, and phasic velocities. Los Alamos was asked to investigate why this difference exists between signal-variable values and their component-data values in the TRCOUT and TRCGRF files of TRAC-P.

\subsection{TESTING}

Looking at the plotted results, we suspected the signal-variable values lead their component-data values because the signal variables are defined at the beginning of the timestep, and the component-data values are defined at the end of the timestep for a given end-of-timestep problem time output edit. This would cause the observed difference between values. The observed divergence between the signal variable values and their component-data values at the end of the problem could be due to this beginning/end-of-timestep defining difference, plotting only even- or odd-numbered timestep results and timestep oscillatory divergence in the numerical solution after being evaluated with a timestep of $\sim 100 \mathrm{~s}$ at a material Courant number of 1000 for $>8000 \mathrm{~s}$ of problem time. 


\section{TABLE I \\ SIGNAL-VARIABLE INPUT DATA ADDED TO THE TRACIN FILE FOR TEST PROBLEM MST2}

\begin{tabular}{|c|c|c|c|c|c|}
\hline IDSV & ISVN & ICLN & ICN1 & ICN2 & \\
\hline 401 & 21 & 4 & 1 & 0 & * Pressure PIPE 4, Cell 1 \\
\hline 402 & 22 & 4 & 1 & 0 & * Vapor Temperature PIPE 4 , Cell 1 \\
\hline 403 & 23 & 4 & 1 & 0 & * Liquid Temperature PIPE 4, Cel1 1 \\
\hline 404 & 27 & 4 & 1 & 0 & * Gas Volume Fraction PIPE 4, Cell 1 \\
\hline 405 & 29 & 4 & 1 & 0 & * Gas Mass Flow PIPE 4, Interface 1 \\
\hline 406 & 32 & 4 & 1 & 0 & * Liquid Mass Flow PIPE 4, Interface 1 \\
\hline 407 & 35 & 4 & 1 & 0 & * Gas Velocity PIPE 4, Interface 1 \\
\hline 408 & 38 & 4 & 1 & 0 & * Liquid Velocity PIPE 4, Interface 1 \\
\hline 409 & 69 & 4 & 1 & 0 & * Mixture Mass Flow PIPE 4, Interface 1 \\
\hline 415 & 29 & 4 & 2 & 0 & * Gas Mass Flow PIPE 4, Interface 2 \\
\hline 416 & 32 & 4 & 2 & 0 & * Liquid Mass Flow PIPE 4, Interface 2 \\
\hline 417 & 35 & 4 & 2 & 0 & * Gas Velocity PIPE 4, Interface 2 \\
\hline 418 & 38 & 4 & 2 & 0 & * Liquid Velocity PIPE 4, Interface 2 \\
\hline 419 & 69 & 4 & 2 & 0 & * Mixture Mass Flow PIPE 4, Interface 2 \\
\hline
\end{tabular}

These suspicions were verified by evaluating the MST2 test problem with output edits to the TRCOUT and TRCGRF files every timestep for timesteps $1(0.0 \mathrm{~s})$ to 25 $(0.0477 \mathrm{~s})$, and $315(9023.1 \mathrm{~s})$ to $325(10024.7 \mathrm{~s})$. The signal-variable and component 4 data output to the TRCOUT file for timesteps 5 to 12,20 to 25 , and 315 to 325 is shown in Appendix A. The parameter values of interest are shown in bold type in the timestep 5 output. These values for timesteps 5 to 12, 20 to 25, and 315 to 325 are tabulated in Table II for signal variables 401 to 408 and their corresponding component 4 data.

Note that in Table II all beginning-of-timestep signal-variable values for a given timestep are the same as their end-of-timestep component-data values from the previous timestep. Their values are consistent for the same point in time. The TRCGRF file plots signal variables at the end-of-timestep time even though they are defined at the beginning-of-timestep time. This causes their values to be shifted one timestep forward making them appear to be inconsistent with their component data.

The MST2 test problem was evaluated a second time with TRCGRF-file graphic edits every timestep for problem times $0 \mathrm{~s}$ to $4 \mathrm{~s}$ and $7000 \mathrm{~s}$ to $10,000 \mathrm{~s}$. Figures 1 to 7 show the one timestep advance of the signal-variable values in the plotted graphics output from TRAC-P. If the signal-variable values were shifted back one timestep in time, their plotted values would be the same as their component-data values.

Plotting parameter values from every timestep for problem times 7000 to $10,000 \mathrm{~s}$ shows oscillatory timestep divergence in Figs. 4 to 7 for the gas volume fraction, phasic and total mass flows, and phasic velocities. If only even- or odd-numbered timesteps were plotted, divergence between the signal-variable and component-data values would appear to be occurring. Again, the signal variables would have the same values as their component data in these lower-half plots if the signal-variable 
values were shifted back one timestep in time. Their plotted values that should be the same are slightly different because of round-off error in the component-data values that are packed four values per storage word vs signal-variable values that are stored one value per storage word in the TRCGRF file.

It is interesting to note that the magnitude of the Cray-evaluated oscillatory timestep divergence evaluated at KAPL on Version 5.4.15 of TRAC-P is 25 times larger than that which was evaluated on an Ultra SUN workstation with Version 5.4.28. Is this due to machine dependence or to corrections and improvements made to TRAC-P between these two versions? To determine if the oscillatory divergence is due to recent changes to TRAC-P, the MST2 test problem, with added signal variables and consecutive timestep edits, was evaluated on a Cray YMP computer with TRAC-P Version 5.3.01 (dated 8/11/92). Figures 8 to 12 show the end-of-problem oscillatory timestep divergence that was evaluated for the gas volume fraction, phasic mass flows, and phasic velocities. Its oscillatory-divergence magnitude is 3.5 times greater than the Cray-evaluated oscillatory divergence at KAPL. Thus, this oscillatory timestep divergence evaluated at a material Courant number of 1000 in test problem MST2 has existed since TRAC-PF1/MOD2 Version 5.3. Its magnitude is believed to have decreased because of corrections and improvements made to TRAC.

A standard test problem should not have oscillatory divergence in its numerical solution. The oscillatory divergence can be eliminated by changing the MST2 test problem end time from 10,000 to $7000 \mathrm{~s}$ or less. It also can be eliminated by evaluating TRAC-P with a maximum material Courant number $<1000$. The later was demonstrated with the DPTCON update in TRAC-P Version 5.4.28 by inputting a one-dimensional maximum material Courant number of 500 and evaluating the MST2 tesi problem for $50,000 \mathrm{~s}$. This reduced the timestep size from $\sim 100$ to $\sim 50 \mathrm{~s}$, and the steady-state solution converged to a constant condition without oscillation or divergence.

\subsection{CONCLUSIONS}

TRAC-P outputs signal variables and their component data to the TRCOUT and TRCGRF files with beginning-of-timestep values for the signal variables and end-oftimestep values for the component data at the end-of-timestep edit time. This shifts the signal-variable values forward in time by one timestep making their values inconsistent with their component-data values in a specific-time output edit. This inconsistency was noted at KAPL and explained in this report by the above statement. Further divergence of signal-variable values and their component-data values after $9000 \mathrm{~s}$ in standard test problem MST2 also was observed at KAPL. This was explained as being due to oscillatory timestep divergence of the numerical solution evaluated with a material Courant number of 1000 when plotted at only even- or odd-numbered timesteps. The oscillatory behavior in problem MST2 was shown to exist in TRAC-PF1/MOD2 Version 5.3.01 as well as in TRAC-P Versions 5.4.15 and 5.4.28. The magnitude of the oscillatory divergence was 25 times greater in Version 5.4.15, and $87(25 \times 3.5)$ times greater in Version 5.3.01 than in the current Version 5.4.28 of TRAC-P. Reducing the maximum material Courant number from 1000 to 500 eliminated the oscillatory-divergence behavior of the numericalsolution in the MST2 test problem. 


\section{ACKNOWLEDGMENT}

The author wishes to thank Richard J. Smith for valuable discussions during the development and testing of this TRAC-P update. 
TABLE II

\section{TEST PROBLEM MST2 PARAMETER VALUES DEFINED BY COMPONENT 4 IN CELL OR INTERFACE 1 AND BY SIGNAL VARIABLES AT TIMESTEPS 5-12, 20-25, AND 315-325}

\begin{tabular}{cccccc} 
Time- & \multicolumn{2}{c}{ - - Pressure (MPa) - - } & \multicolumn{2}{c}{ Gas Temperature (K) - } \\
step & Time & Sig. Var. & Comp. 4 & Sig. Var. & Comp. 4 \\
Number & $(\mathrm{s})$ & 401 & Cell 1 & 402 & Cell 1 \\
5 & & & & & \\
6 & 0.0055 & 1.064938 & 1.06527 & 460.6876 & 460.4 \\
7 & 0.0068 & 1.065269 & 1.06533 & 460.3781 & 460.0 \\
8 & 0.0081 & 1.065332 & 1.06532 & 460.0459 & 459.7 \\
9 & 0.0095 & 1.065317 & 1.06528 & 459.7122 & 459.4 \\
10 & 0.0110 & 1.065280 & 1.06524 & 459.3839 & 459.1 \\
11 & 0.0126 & 1.065236 & 1.06519 & 459.0634 & 458.8 \\
12 & 0.0142 & 1.065189 & 1.06514 & 458.7519 & 458.5 \\
20 & 0.0159 & 1.065142 & 1.06510 & 458.4506 & 458.2 \\
21 & 0.0331 & 1.064787 & 1.06473 & 456.4773 & 456.3 \\
22 & 0.0357 & 1.064732 & 1.06467 & 456.2895 & 456.1 \\
23 & 0.0385 & 1.064674 & 1.06461 & 456.1152 & 456.0 \\
24 & 0.0414 & 1.064611 & 1.06454 & 455.9539 & 455.8 \\
25 & 0.0445 & 1.064540 & 1.06446 & 455.8054 & 455.7 \\
315 & 0.0477 & 1.064461 & 1.06437 & 455.6690 & 455.5 \\
316 & 9023.1 & 1.108477 & 1.10848 & 456.6253 & 456.6 \\
317 & 9123.3 & 1.108477 & 1.10848 & 456.6253 & 456.6 \\
318 & 9223.4 & 1.108477 & 1.10848 & 456.6253 & 456.6 \\
319 & 9323.6 & 1.108477 & 1.10848 & 456.6253 & 456.6 \\
320 & 9423.7 & 1.108477 & 1.10848 & 456.6253 & 456.6 \\
321 & 9523.9 & 1.108477 & 1.10848 & 456.6253 & 456.6 \\
322 & 9624.0 & 1.108477 & 1.10848 & 456.6253 & 456.6 \\
323 & 9724.2 & 1.108477 & 1.10848 & 456.6253 & 456.6 \\
324 & 9924.4 & 1.108477 & 1.10848 & 456.6253 & 456.6 \\
325 & 10024.7 & 1.108477 & 1.10848 & 456.6253 & 456.6 \\
& & 1.108477 & 1.10848 & 456.6253 & 456.6 \\
& 9924.5 & & & &
\end{tabular}




\section{TABLE II (Cont)}

\section{TEST PROBLEM MST2 PARAMETER VALUES DEFINED BY COMPONENT 4 IN CELL OR INTERFACE 1 AND BY SIGNAL VARIABLES AT TIMESTEPS 5-12, 20-25, AND 315-325}

\begin{tabular}{|c|c|c|c|c|c|}
\hline \multirow{3}{*}{$\begin{array}{l}\text { Time- } \\
\text { step } \\
\text { Number }\end{array}$} & \multirow{3}{*}{$\begin{array}{c}\text { Time } \\
\text { (s) }\end{array}$} & \multicolumn{2}{|c|}{-Liquid Temperature (K) } & \multicolumn{2}{|c|}{ Gas Volume Fraction (-) } \\
\hline & & Sig. Var. & Comp. 4 & Sig. Var. & Comp. 4 \\
\hline & & 403 & Cell 1 & 404 & Cell 1 \\
\hline 5 & 0.0055 & 454.8754 & 454.9 & 0.5002954 & 0.5003 \\
\hline 6 & 0.0068 & 454.8727 & 454.9 & 0.5003280 & 0.5004 \\
\hline 7 & 0.0081 & 454.8705 & 454.9 & 0.5003681 & 0.5004 \\
\hline 8 & 0.0095 & 454.8684 & 454.9 & 0.5004169 & 0.5005 \\
\hline 9 & 0.0110 & 454.8663 & 454.9 & 0.5004752 & 0.5005 \\
\hline 10 & 0.0126 & 454.8641 & 454.9 & 0.5005437 & 0.5006 \\
\hline 11 & 0.0142 & 454.8619 & 454.9 & 0.5006229 & 0.5007 \\
\hline 12 & 0.0159 & 454.8596 & 454.9 & 0.5007135 & 0.5008 \\
\hline 20 & 0.0331 & 454.8415 & 454.8 & 0.5019179 & 0.5021 \\
\hline 21 & 0.0357 & 454.8391 & 454.8 & 0.5021377 & 0.5024 \\
\hline 22 & 0.0385 & 454.8367 & 454.8 & 0.5023751 & 0.5026 \\
\hline 23 & 0.0414 & 454.8341 & 454.8 & 0.5026315 & 0.5029 \\
\hline 24 & 0.0445 & 454.8314 & 454.8 & 0.5029090 & 0.5032 \\
\hline 25 & 0.0477 & 454.8286 & 454.8 & 0.5032107 & 0.5035 \\
\hline 315 & 9023.1 & 456.4913 & 456.5 & 0.3328429 & 0.3328 \\
\hline 316 & 9123.3 & 456.4913 & 456.5 & 0.3328388 & 0.3328 \\
\hline 317 & 9223.4 & 456.4913 & 456.5 & 0.3328440 & 0.3328 \\
\hline 318 & 9323.6 & 456.4913 & 456.5 & 0.3328373 & 0.3328 \\
\hline 319 & 9423.7 & 456.4913 & 456.5 & 0.3328459 & 0.3328 \\
\hline 320 & 9523.9 & 456.4913 & 456.5 & 0.3328349 & 0.3328 \\
\hline 321 & 9624.0 & 456.4913 & 456.5 & 0.3328490 & 0.3328 \\
\hline 322 & 9724.2 & 456.4913 & 456.5 & 0.3328310 & 0.3329 \\
\hline 323 & 9824.4 & 456.4913 & 456.5 & 0.3328540 & 0.3328 \\
\hline 324 & 9924.5 & 456.4913 & 456.5 & 0.3328247 & 0.3329 \\
\hline 325 & 10024.7 & 456.4913 & 456.5 & 0.3328622 & 0.3328 \\
\hline
\end{tabular}


TABLE II (Cont)

\section{TEST PROBLEM MST2 PARAMETER VALUES DEFINED BY COMPONENT 4 IN CELL OR INTERFACE 1 AND BY SIGNAL VARIABLES AT TIMESTEPS 5-12, 20-25, AND 315-325}

\begin{tabular}{cccccc}
$\begin{array}{c}\text { Time- } \\
\text { step }\end{array}$ & Time & \multicolumn{2}{c}{ Gas Mass Flow $(\mathrm{kg} / \mathrm{s})$ - } & - Liquid Mass Flow $(\mathrm{kg} / \mathrm{s})$ \\
Number & $(\mathrm{s})$ & 405 & Cell 1 & Sig. Var. & Comp. 4 \\
5 & 0.0055 & -0.4128925 & -0.4431 & -23.79867 & -31.54 \\
6 & 0.0068 & -0.4430732 & -0.4694 & -31.54327 & -39.29 \\
7 & 0.0081 & -0.4693900 & -0.4953 & -39.29019 & -47.05 \\
8 & 0.0095 & -0.4953126 & -0.5215 & -47.04938 & -54.84 \\
9 & 0.0110 & -0.5215492 & -0.5475 & -54.83852 & -62.66 \\
10 & 0.0126 & -0.5475279 & -0.5722 & -62.66484 & -70.52 \\
11 & 0.0142 & -0.5722365 & -0.5944 & -70.52449 & -78.41 \\
12 & 0.0159 & -0.5944209 & -0.6124 & -78.40673 & -86.30 \\
20 & 0.0331 & -0.6808401 & -0.7213 & -143.2853 & -151.8 \\
21 & 0.0357 & -0.7213369 & -0.7681 & -151.7519 & -160.5 \\
22 & 0.0385 & -0.7680551 & -0.8230 & -160.4573 & -169.6 \\
23 & 0.0414 & -0.8230028 & -0.8884 & -169.5725 & -179.3 \\
24 & 0.0445 & -0.8884351 & -0.9662 & -179.3224 & -190.0 \\
15 & 0.0477 & -0.9661574 & -1.057 & -189.9507 & -201.7 \\
315 & 9023.1 & 2.543331 & 2.544 & 701.7245 & 702.0 \\
316 & 9123.3 & 2.544103 & 2.543 & 701.9616 & 701.7 \\
317 & 9223.4 & 2.543116 & 2.544 & 701.6583 & 702.0 \\
318 & 9323.6 & 2.544378 & 2.543 & 702.0461 & 701.6 \\
319 & 9423.7 & 2.542764 & 2.545 & 701.5502 & 702.2 \\
320 & 9523.9 & 2.544828 & 2.542 & 702.1843 & 701.4 \\
321 & 9624.0 & 2.542189 & 2.546 & 701.3735 & 702.4 \\
322 & 9724.2 & 2.545563 & 2.541 & 702.4103 & 701.1 \\
323 & 9824.4 & 2.541249 & 2.547 & 701.0844 & 702.8 \\
324 & 9924.5 & 2.546764 & 2.540 & 702.7797 & 700.6 \\
325 & 10024.7 & 2.539711 & 2.549 & 700.6117 & 703.4
\end{tabular}


TABLE II (Cont)

\section{TEST PROBLEM MST2 PARAMETER VALUES DEFINED BY COMPONENT 4 IN CELL OR INTERFACE 1 AND BY SIGNAL VARIABLES AT TIMESTEPS 5-12, 20-25, AND 315-325}

\begin{tabular}{|c|c|c|c|c|c|}
\hline Time- & & - - Gas Velo & $y(m / s)--$ & - - Liquid Velc & g (1010) \\
\hline $\begin{array}{l}\text { step } \\
\text { Number }\end{array}$ & $\begin{array}{l}\text { Time } \\
(s)\end{array}$ & $\begin{array}{l}\text { Sig. Var. } \\
\quad 407\end{array}$ & $\begin{array}{l}\text { Comp. } 4 \\
\text { Cell } 1\end{array}$ & $\begin{array}{l}\text { Sig. Var. } \\
\quad 408\end{array}$ & $\begin{array}{l}\text { Comp. } 4 \\
\text { Cell } 1\end{array}$ \\
\hline 5 & 0.0055 & -0.1609021 & -0.1724 & -0.05380208 & -0.07131 \\
\hline 6 & 0.0068 & -0.1724383 & -0.1825 & -0.07131485 & -0.08884 \\
\hline 7 & 0.0081 & -0.1824792 & -0.1924 & -0.08883642 & -0.1064 \\
\hline 8 & 0.0095 & -0.1923560 & -0.2023 & -0.1063903 & -0.1240 \\
\hline 9 & 0.0110 & -0.2023375 & -0.2122 & -0.1240176 & -0.1417 \\
\hline 10 & 0.0126 & -0.2122001 & -0.2216 & -0.1417360 & -0.1595 \\
\hline 11 & 0.0142 & -0.2215521 & -0.2299 & -0.1595379 & -0.1774 \\
\hline 12 & 0.0159 & -0.2299100 & -0.2366 & -0.1774005 & -0.1953 \\
\hline 20 & 0.0331 & -0.2612941 & -0.2766 & -0.3249693 & -0.3443 \\
\hline 21 & 0.0357 & -0.2765786 & -0.2942 & -0.3443223 & -0.3642 \\
\hline 22 & 0.0385 & -0.2942200 & -0.3150 & -0.3642474 & -0.3851 \\
\hline 23 & 0.0414 & -0.3149800 & -0.3397 & -0.3851366 & -0.4075 \\
\hline 24 & 0.0445 & -0.3397118 & -0.3691 & -0.4075069 & -0.4319 \\
\hline 25 & 0.0477 & -0.3690922 & -0.4033 & -0.4319201 & -0.4588 \\
\hline 315 & 9023.1 & 1.350955 & 1.351 & 1.212017 & 1.212 \\
\hline 316 & 9123.3 & 1.351365 & 1.351 & 1.212427 & 1.212 \\
\hline 317 & 9223.4 & 1.350841 & 1.352 & 1.211903 & 1.213 \\
\hline 318 & 9323.6 & 1.351511 & 1.351 & 1.212573 & 1.212 \\
\hline 319 & 9423.7 & 1.350654 & 1.352 & 1.211716 & 1.213 \\
\hline 320 & 9523.9 & 1.351750 & 1.350 & 1.212811 & 1.211 \\
\hline 321 & 9624.0 & 1.350349 & 1.352 & 1.211411 & 1.213 \\
\hline 322 & 9724.2 & 1.352141 & 1.350 & 1.213202 & 1.211 \\
\hline 323 & 9824.4 & 1.349849 & 1.353 & 1.210912 & 1.214 \\
\hline 324 & 9924.5 & 1.352779 & 1.349 & 1.213840 & 1.210 \\
\hline 325 & 10024.7 & 1.349032 & 1.354 & 1.210095 & 1.215 \\
\hline
\end{tabular}



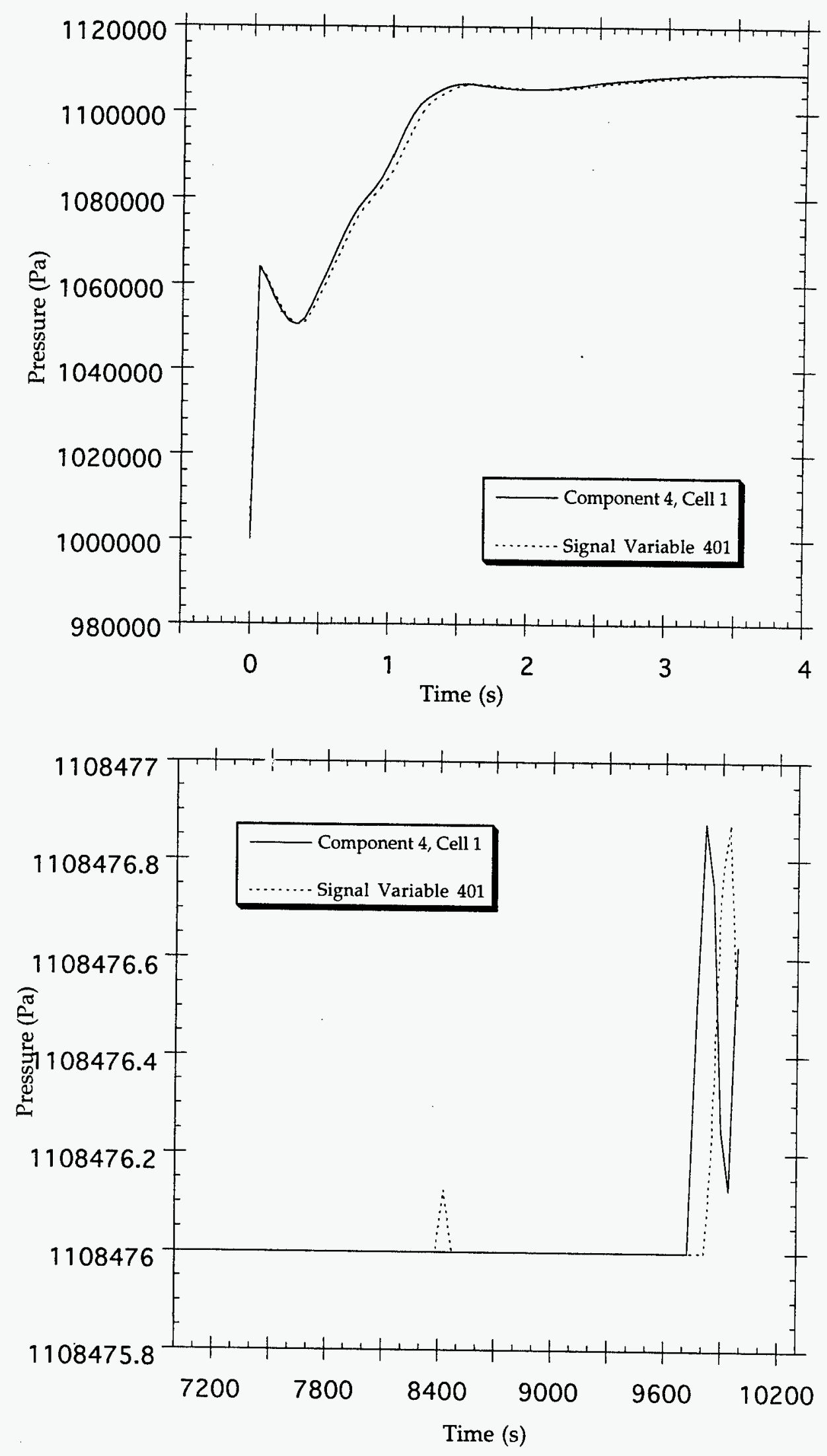

Fig. 1. Pressure vs time ( 0 to $4 \mathrm{~s}$ and 7000 to $10000 \mathrm{~s}$ ) in component 4 , cell 1 and as defined by signal variable 401 . 

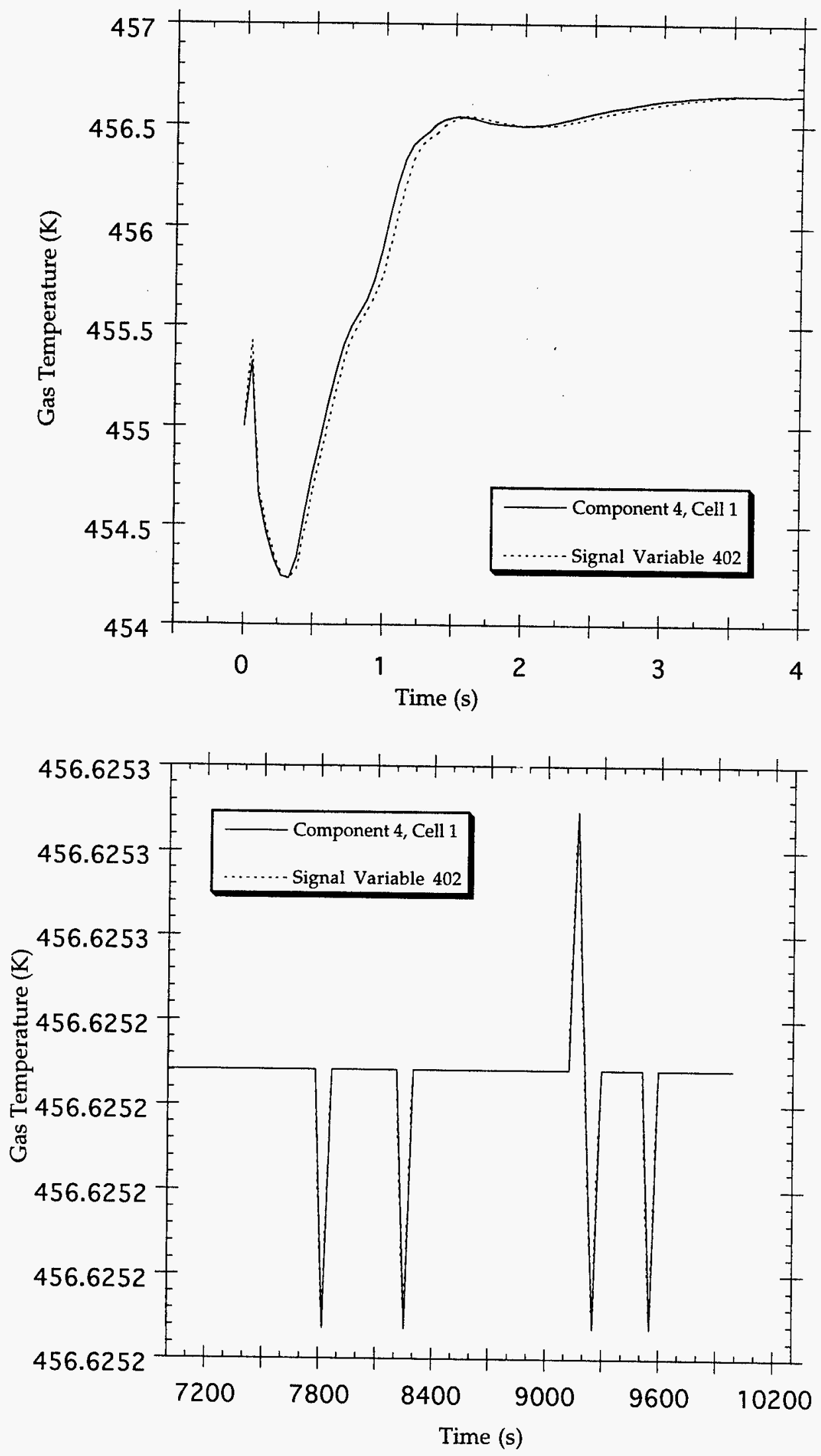

Fig. 2. Gas temperature vs time ( 0 to $4 \mathrm{~s}$ and 7000 to $10000 \mathrm{~s}$ ) in component 4 , cell 1 and as defined by signal variable 402 . 

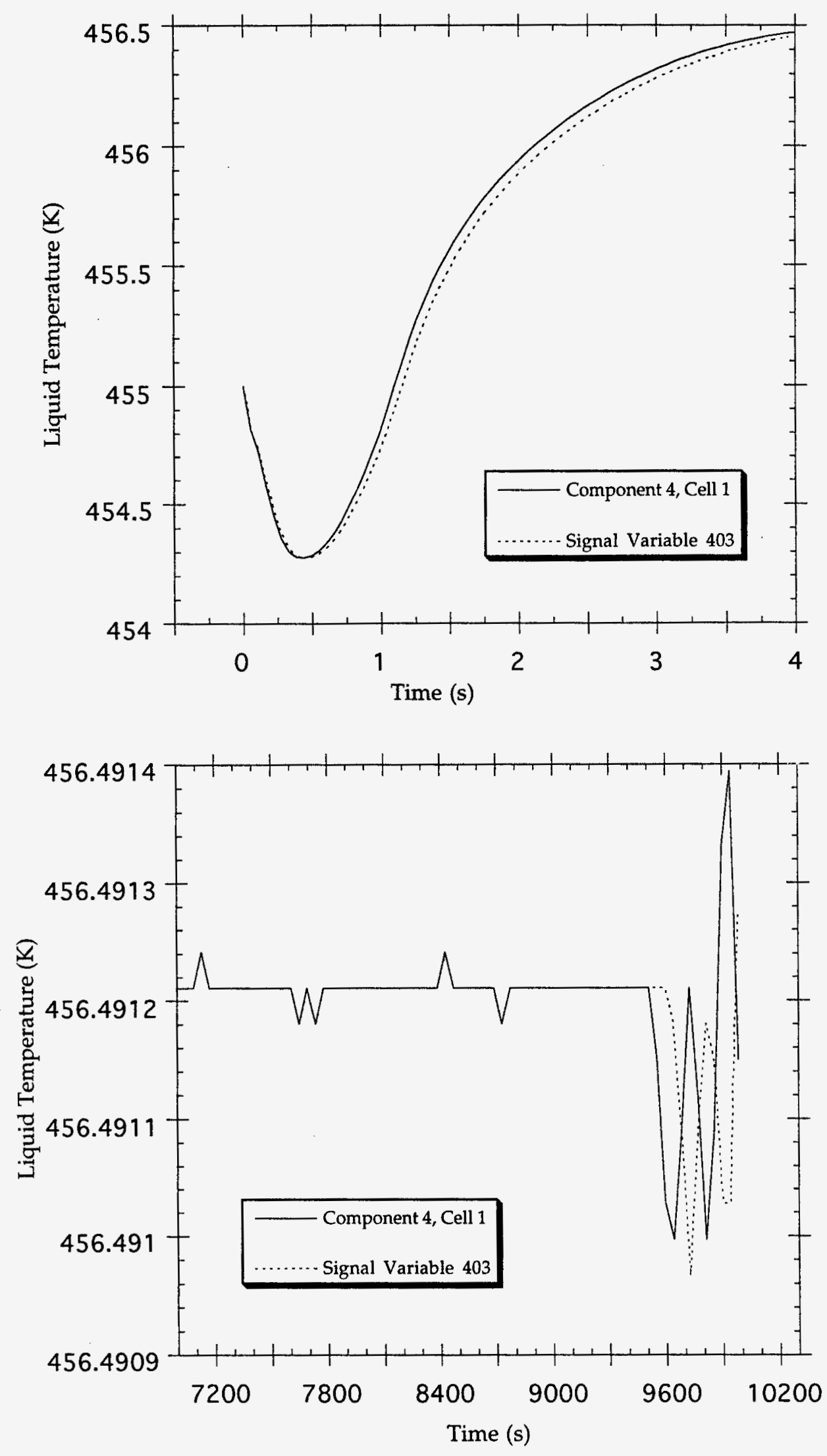

Fig. 3. Liquid temperature vs time ( 0 to $4 \mathrm{~s}$ and 7000 to $10000 \mathrm{~s}$ ) in component 4 , cell 1 and as defined by signal variable 403 . 

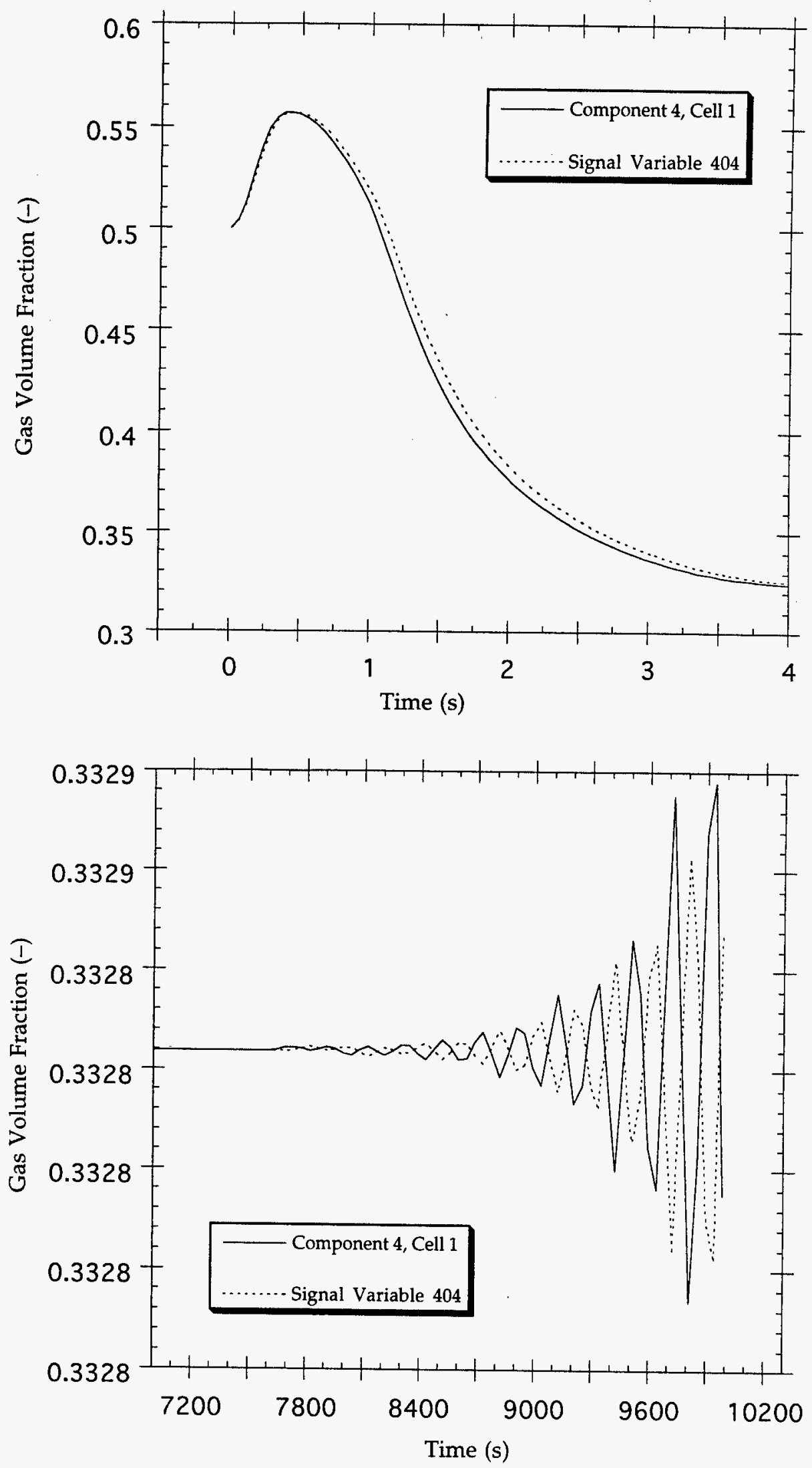

Fig. 4. Gas volume fraction vs time (0 to 4 and 7000 to $10000 \mathrm{~s})$ in component 4 , cell 1 and as defined by signal variable 404 . 

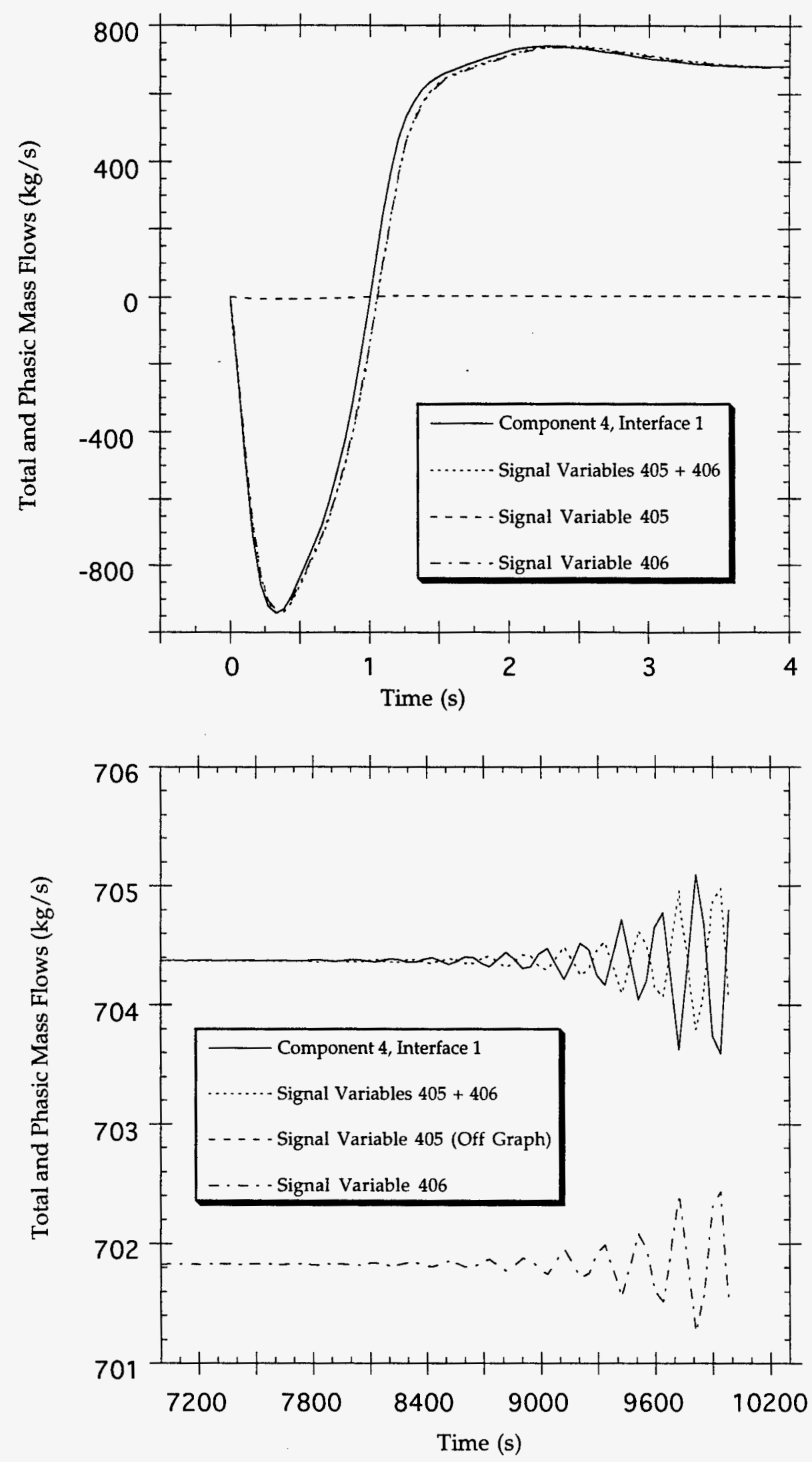

Fig. 5. Mass flows vs time ( 0 to $4 \mathrm{~s}$ and 7000 to $10000 \mathrm{~s}$ ) at component 4 , interface 1 and as defined by signal variables 405 and 406 . 

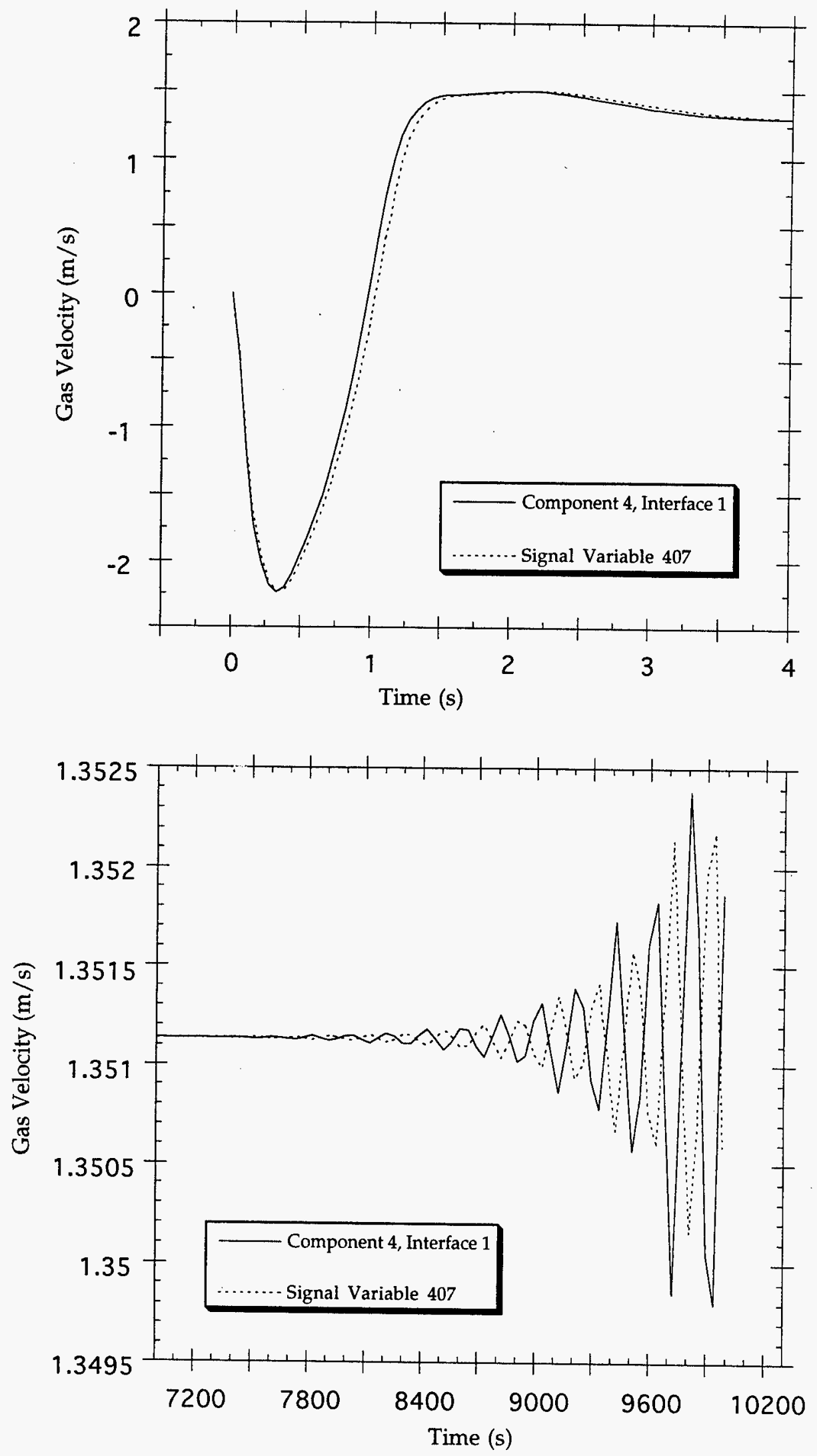

Fig. 6. Gas velocity vs time ( 0 to $4 \mathrm{~s}$ and 7000 to $10000 \mathrm{~s}$ ) at component 4 , interface 1 and as defined by signal variable 407 . 

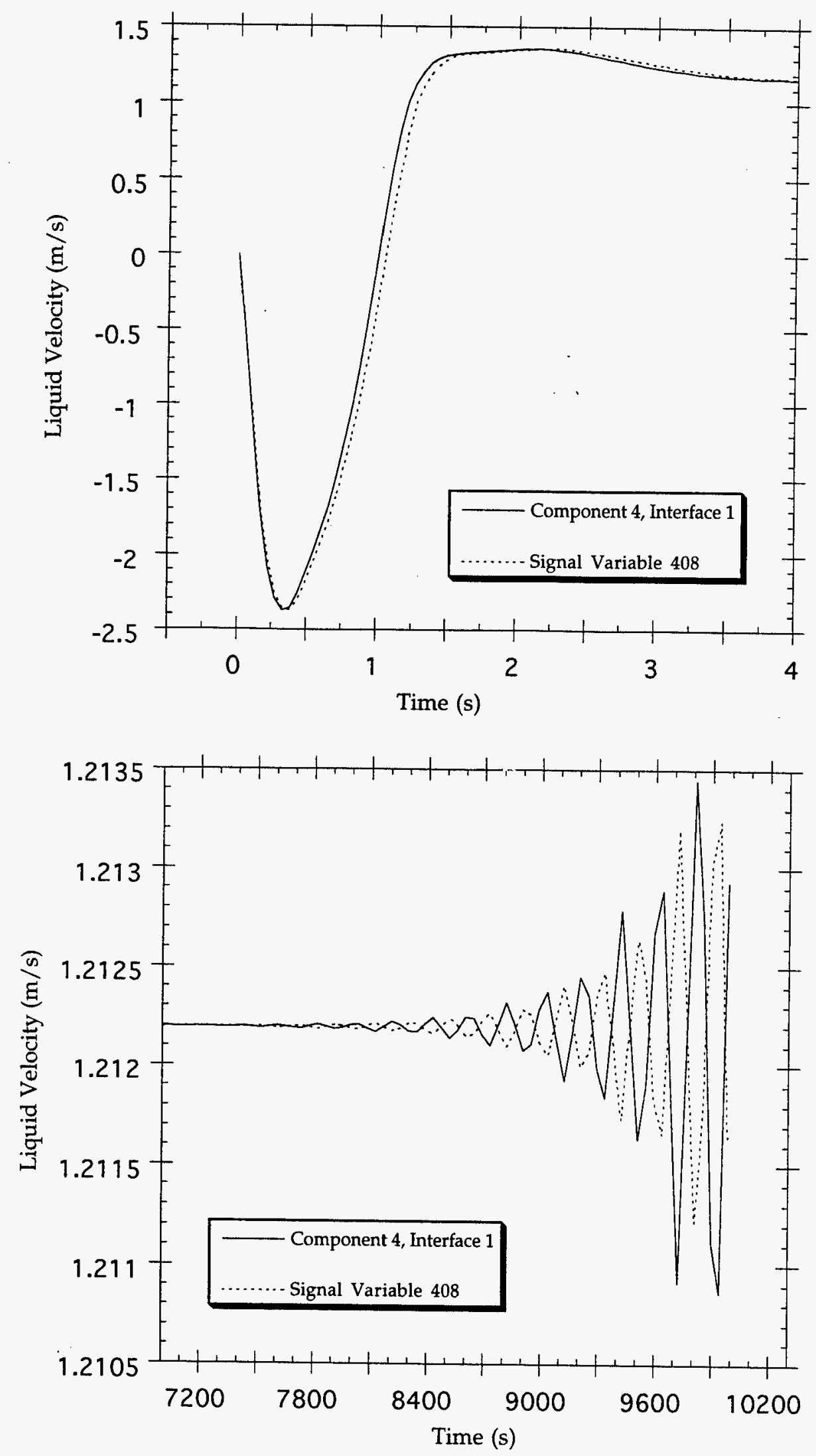

Fig. 7. Liquid velocity vs time ( 0 to $4 \mathrm{~s}$ and 7000 to $10000 \mathrm{~s}$ ) at component 4 , interface 1 and as defined by signal variable 408 . 


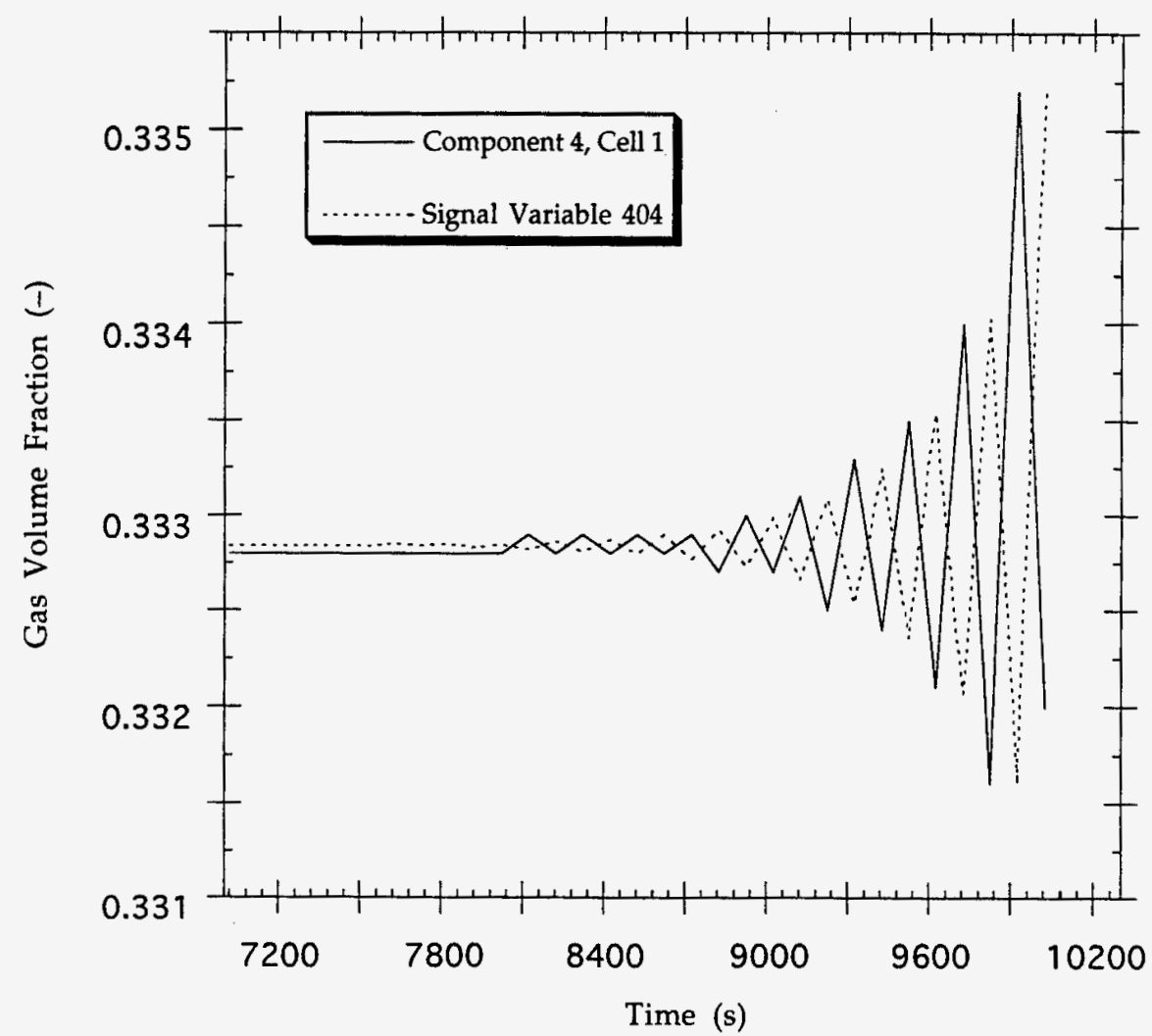

Fig. 8. Gas volume fraction vs time in component 4, cell 1 , and as defined by signal variable 404 for Version 5.3.01.

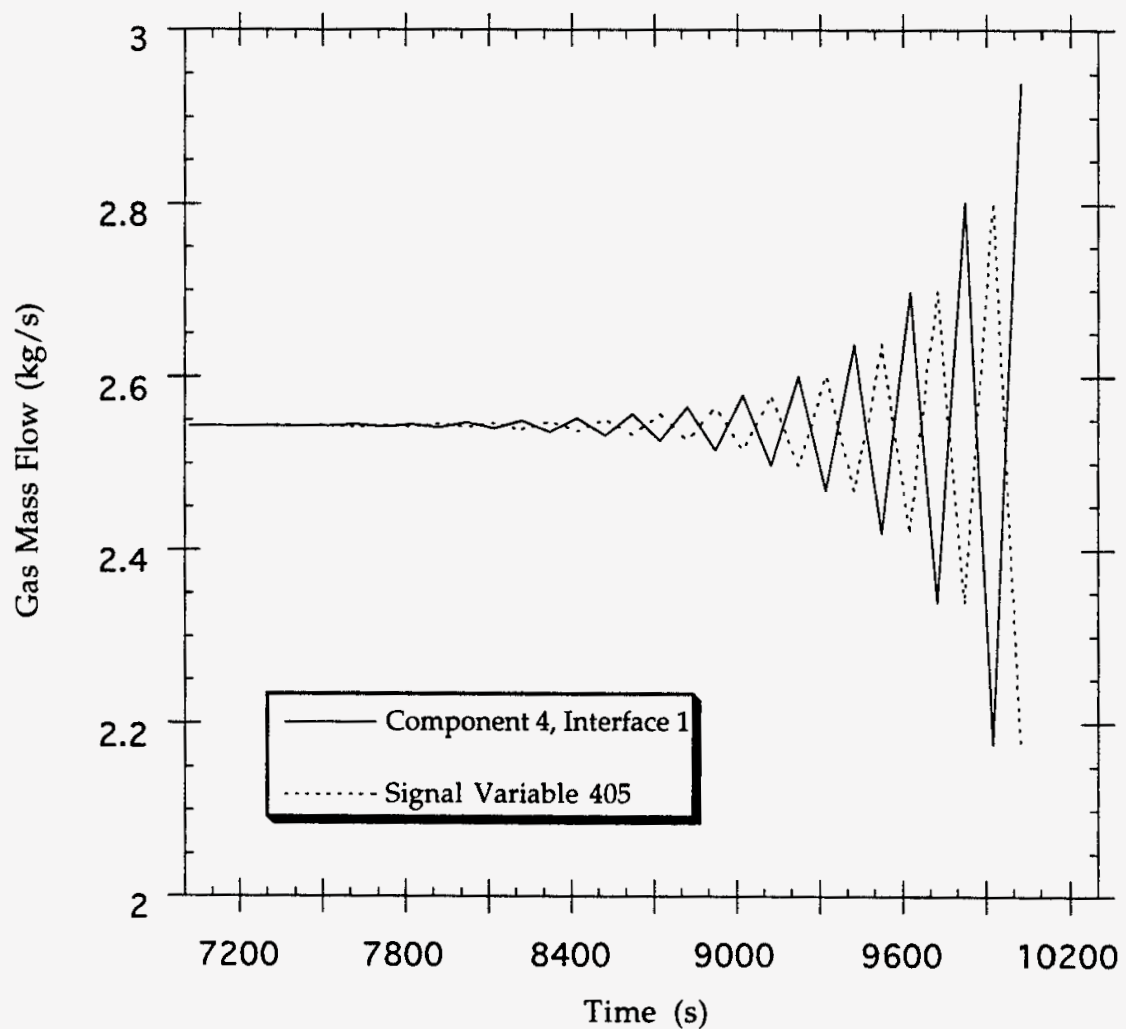

Fig. 9. Gas mass flow vs time in component 4 , interface 1 and as defined by signal variable 405 for Version 5.3.01. 


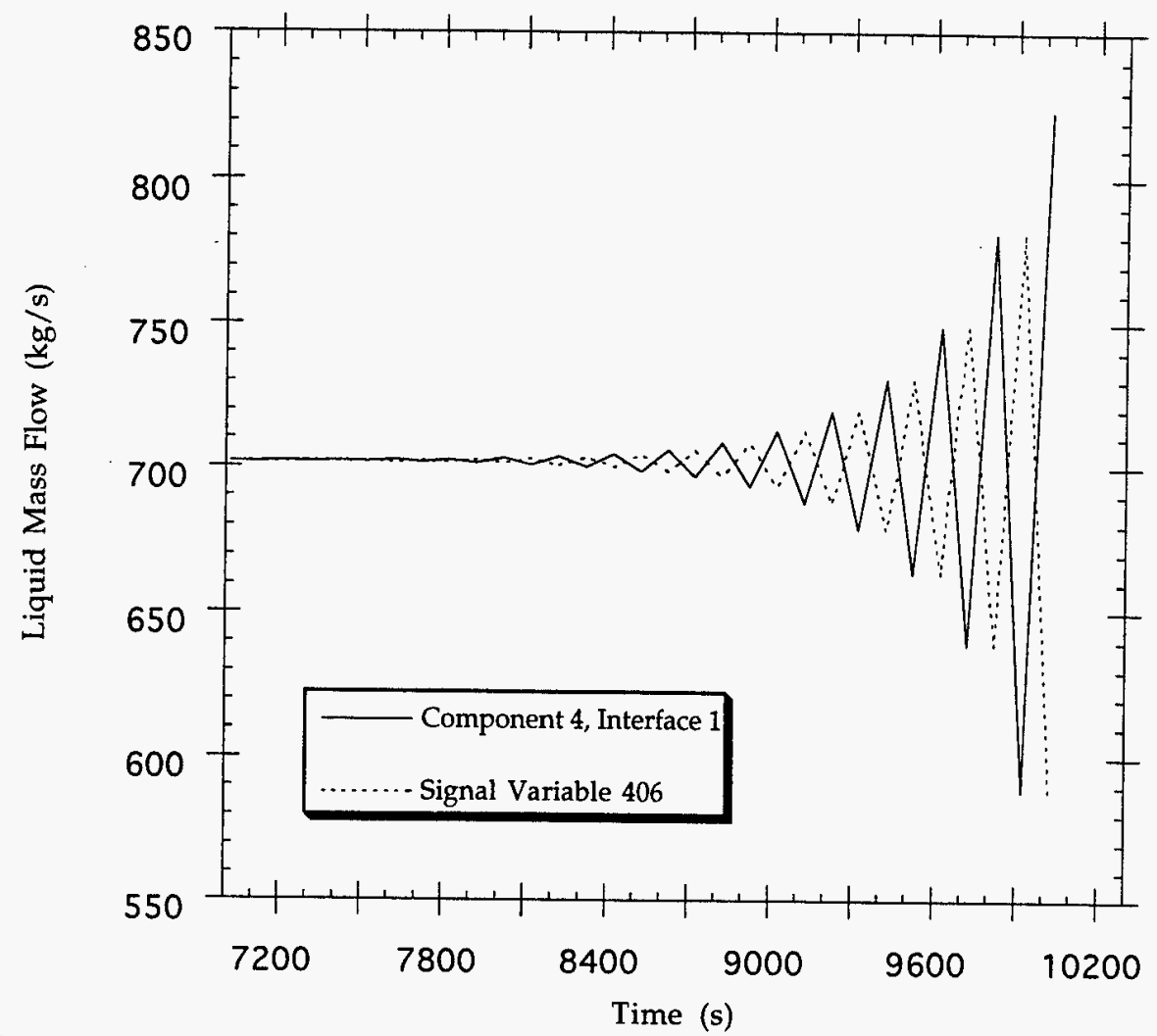

Fig. 10. Liquid mass flow vs time in component 4 , interface 1 and as defined by signal variable 406 for Version 5.3.01.

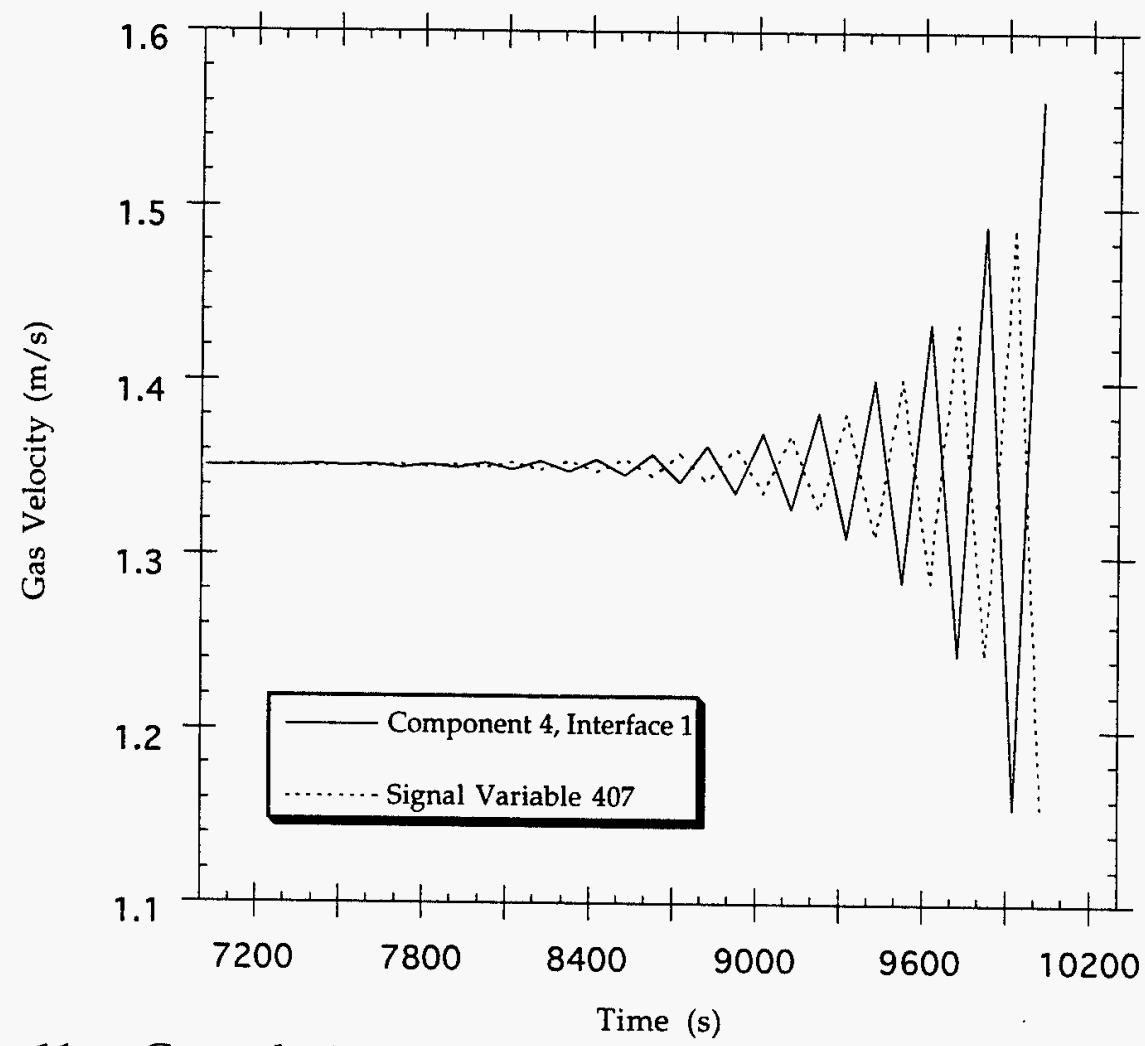

Fig. 11. Gas velocity vs time in component 4 , interface 1 and as defined by signal variable 407 for Version 5.3.01. 


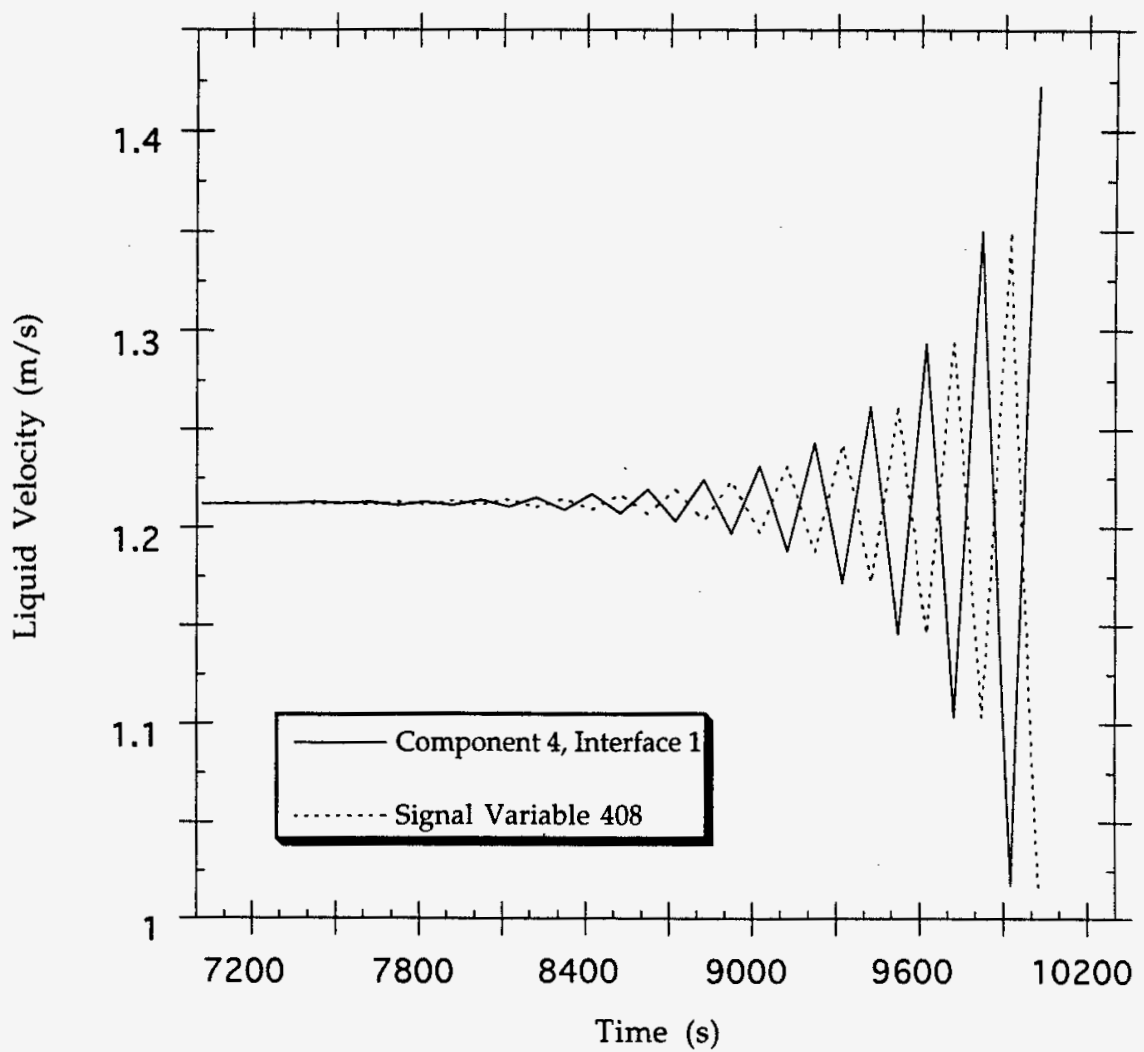

Fig. 12. Liquid velocity vs time in component 4 , interface 1 and as defined by signal variable 408 for Version 5.3.01. 


\section{Appendix A \\ Test problem MST2 \\ Large edits every timestep from timesteps 5 to 12,20 to 25 and 315 to 325 .}

3191

3192

3193

3217

3218

3219

3220

3221

3222

3223
3224

3225

3226

3259

3260

3261

3262
3263

3264

3265

3266

3268

3269

3270

3271

3273

3274

4018

4019

4020

4021

4045

4046

4047

4048

4050

4051

4052

4053

4087

4087

4088

4090

4091
4092

4093

4094

4095

4096

4097

4098
4099

4100

4101

4845

4847

4848

4871

4872

4874

4875

4876

4878

4878

4880

4913

4914
4915

4916

4917

4918

4919

4920

4921
4922

4923

4924

4925

4926 trac large edit

problem time is 5.525631E-03 $\mathrm{s}$, time-step size is $1.2155 \mathrm{E}-03 \mathrm{~s}$, time-step number is

5, outer-iteration number is $\cdot 2$

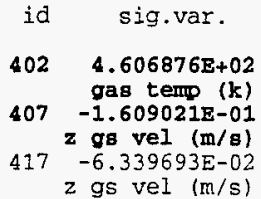

0.00431

id sig.var.

$403 \quad 4.548754 \mathrm{E}+02$

$408 \quad-5.380208 \mathrm{E}-02$

2 lq vel (m/s)

$418-4.270380 \mathrm{E}-02$

2 lq vel (m/s) id sig.var.

$404 \quad 5.002954 \mathrm{E}-01$ void fraction

$409-2.421156 \mathrm{E}+01$ $\mathrm{z} \mathrm{mmfw}(\mathrm{kg} / \mathrm{s}$ )

$419-9.526822 \mathrm{E}+00$

$\mathrm{z} \mathrm{m} \mathrm{mfw}(\mathrm{kg} / \mathrm{s})$

the component type is a pipe, component number is 4 , first junction number is 4 , and second junction number is 8

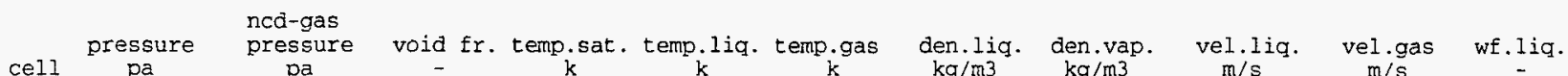

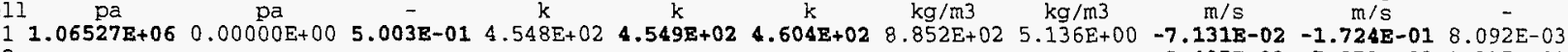
$2 \quad-5.495 \mathrm{E}-02 \quad-7.873 \mathrm{E}-02 \quad 1.335 \mathrm{E}-01$

dis.sol. p.o.sol. Er.new if.drag temp.ss. evap. cond. mfw.liq. mfw.vap. velt.liq velt.gas wf.gas

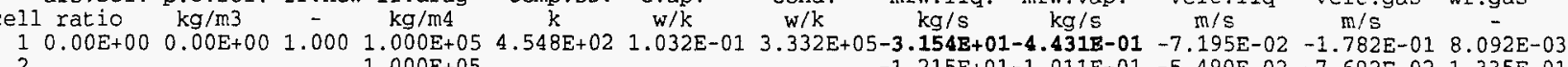

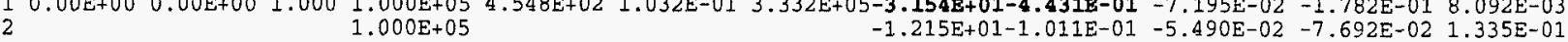

pipe component total coolant mass is $4.44903 \mathrm{E}+02 \mathrm{~kg}$, total coolant energy is $3.46380 \mathrm{E}+08 \mathrm{w}^{\star} \mathrm{s}$, and

computed initial total coolant mass is $4.44938 \mathrm{E}+02 \mathrm{~kg}$

rac large edit

problem time is $6.801913 \mathrm{E}-03 \mathrm{~s}$, time-step size is $1.2763 \mathrm{E}-03 \mathrm{~s}$, time-step number is

6, outer-iteration number is 2

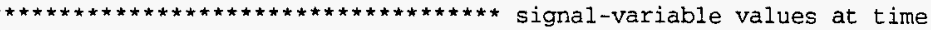

$0.00553 \mathrm{~s}$

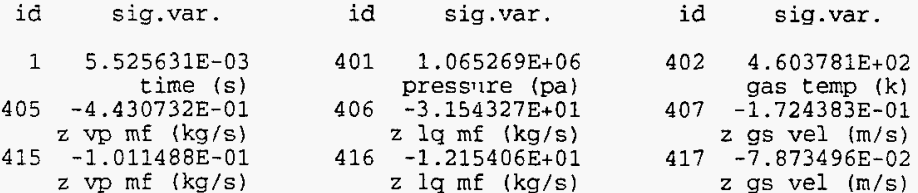

id sig.var.

id sig.var.

$$
\begin{aligned}
& 5.525631 \mathrm{E}-0 \\
& 5-4.430732 \mathrm{E}-0 \\
& \text { z vp } \mathrm{mf}(\mathrm{kg} / \mathrm{s}
\end{aligned}
$$$$
\mathrm{z} \text { lq } \mathrm{mf}(\mathrm{kg} / \mathrm{s})
$$$$
z \text { gs vel }(\mathrm{m} / \mathrm{s})
$$

$403 \quad 4.548727 \mathrm{E}+02$

$408 \quad-7.131485 \mathrm{E}-02$

$408-7.131485 \mathrm{E}-02$

$418 \quad-5.495000 \mathrm{E}-02$

$z$ lq vel $(\pi / s)$
$404 \quad 5.003280 \mathrm{E}-01$

void fraction

$409-3.198634 \mathrm{E}+0$

$2 \mathrm{~m} \mathrm{mfw}(\mathrm{kg} / \mathrm{s})$

$419-1.225521 \mathrm{E}+01$

$2 \mathrm{~m} \mathrm{mfw}(\mathrm{kg} / \mathrm{s})$

the component type is a pipe, component number is 4 , first junction number is 4 , and second junction number is 8

pressure pressure void fr. temp.sat. temp.Iiq. temp.gas den.liq. den.vap. vel.liq. vel.gas wf.liq.

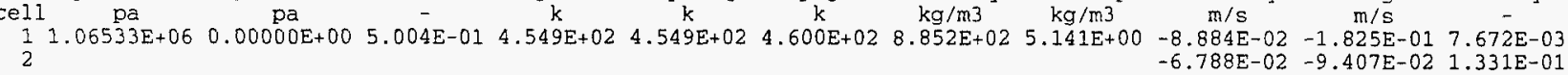

dis.sol. p.o.sol. fr.new if.drag temp.ss. evap. cond. mfw.liq. mfw.vap. velt.liq velt.gas wf.gas

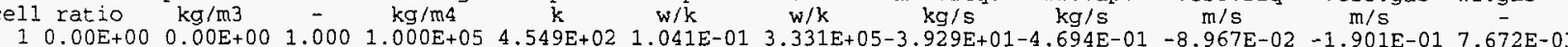

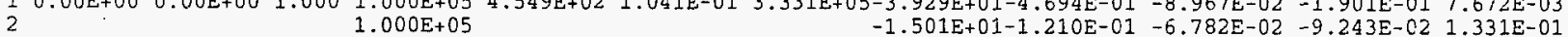

pipe component total coolant mass is $4.44879 \mathrm{E}+02 \mathrm{~kg}$, total coolant energy is $3.46361 \mathrm{E}+08 \mathrm{w*}$, and

computed initial total coolant mass is $4.44938 \mathrm{E}+02 \mathrm{~kg}$

trac large edit

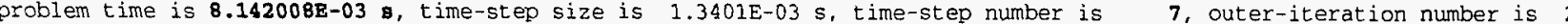

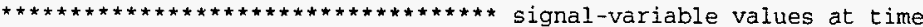

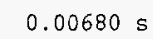

id sig.var.

$16.801913 \mathrm{E}-03$ time (s)

$405-4.693900 \mathrm{E}-01$ $\mathrm{z} \mathrm{vp} \mathrm{mf}(\mathrm{kg} / \mathrm{s})$

$415-1.209759 \mathrm{E}-01$

$\mathrm{z} \mathrm{vp} \mathrm{mf}(\mathrm{kg} / \mathrm{s})$ id sig.var.

$401 \quad 1.065332 \mathrm{E}+06$

406 pressure (pa) 2 $1 \mathrm{~g} \mathrm{mf}(\mathrm{kg} / \mathrm{s})$

$416-1.501296 \mathrm{E}+01$

$\mathrm{z} \mathrm{lq} \mathrm{mf}(\mathrm{kg} / \mathrm{s})$ id sig.var.

$402 \quad 4.600459 E+02$

$407 \quad-1.824792 \mathrm{E}-01$

$z$ gs vel (m/s)

417 - $0.406994 \mathrm{E}-02$

$z$ gs vel (m/s)

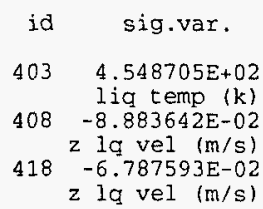

id sig.var.

$404 \quad 5.003681 \mathrm{E}-01$ 409 void Eraction z

$419^{z} \mathrm{~m} \mathrm{mw}(\mathrm{kg} / \mathrm{s}$ z m mfw $(\mathrm{kg} / \mathrm{s})$

the component type is a pipe, component number is 4 , first junction number is 4 , and second junction number is 8 pressure pressure void fr. temp.sat. temp.liq. temp.gas den.liq. den.vap. vel.liq. vel.gas wf.liq.

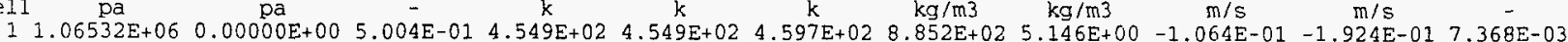

dis.sol. p.o.sol. Er.new if.drag temp.ss. evap.

ew if.drag temp.ss. evap. cond.

$\begin{array}{lllll}1 & 0.00 \mathrm{E}+00 & 0.00 \mathrm{E}+00 & 1.000 & 1.000 \mathrm{E}+05\end{array}$

1. $000 \mathrm{E}+05$

mfw.liq. mfw.vap. $-8.152 \mathrm{E}-02-1.099 \mathrm{E}-01 \quad 1.328 \mathrm{E}-01$ 
4927 pipe component total coolant mass is $4.44848 \mathrm{E}+02 \mathrm{~kg}$, total coolant energy is $3.46336 \mathrm{E}+08 \mathrm{w}^{\star} \mathrm{s}$, and 4928 computed initial total coolant mass is $4.44938 \mathrm{E}+02 \mathrm{~kg}$

5672

5673

5674
5675

5675
5698

5698
5699

5700

5701

5702

5703

5704
5705

5706

5707

5740

5741
5742

5743

5744
5745

5746
5747

5748

5749

5750

5751
5752

5753

5754

5755

6499

6500

6501

6502

6525
6526

6527

6528

6529

6531

6531

6533

6534

6567

6568
6569

6570

6571

6572

6573

6575

6576

6577

6578

5579

6581

6582

7326

7327

7328
7329

7352

7353

7354

7356

7357

7358

7359
7360

7361

7394

7395

7396

7397

7398

7399
7400

7401

7402

7403

7404

7405

7406

7408

7409

rac large edit

problem time is $9.549109 \mathrm{E}-03 \mathbf{8}$, time-step size is $1.4071 \mathrm{E}-03 \mathrm{~s}$, time-step number is $\mathbf{8}$, outer-iteration number is 2

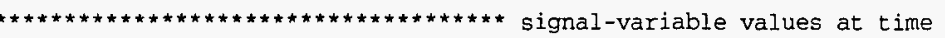
id sig.var.
$18.142008 \mathrm{E}-03$
$405-4.953126 \mathrm{E}-01$
$z \mathrm{vp} \mathrm{mf}(\mathrm{kg} / \mathrm{s})$
$415-1.414088 \mathrm{E}-01$
id sig.var.
$401 \quad 1.065317 \mathrm{E}+06$ pressure (pa)
$406 \quad-4.704938 \mathrm{E}+01$
$416 \quad-1.803024 \mathrm{E}+01$
$\mathrm{z}$ lq $\mathrm{mf}(\mathrm{kg} / \mathrm{s})$

the component type is a pipe, component number is

ncd-gas

1 pressure pressure void fr. temp.sat. temp.liq. temp.gas den.liq. den.vap. vel.liq. vel.gas wf.Iiq. $\begin{array}{lcccccccccccc}1 & 1.06528 \mathrm{E}+06 & 0.00000 \mathrm{E}+00 & 5.005 \mathrm{E}-01 & 4.549 \mathrm{E}+02 & 4.549 \mathrm{E}+02 & 4.594 \mathrm{E}+02 & 8.852 \mathrm{E}+02 & 5.150 \mathrm{E}+00 & -1.240 \mathrm{E}-01 & -2.023 \mathrm{E}-01 & 7.133 \mathrm{E}-03\end{array}$ $\begin{array}{rrr}1.325 \mathrm{E}-01 & -9.592 \mathrm{E}-02-1.263 \mathrm{E}-01 \quad 1.32\end{array}$

dis.sol. p.o.sol. fr.new if.drag temp.ss. evap. cond. $\mathrm{kfw.liq.} \mathrm{mfw.vap.} \mathrm{velt.liq} \mathrm{velt.gas} \mathrm{wf.gas}$

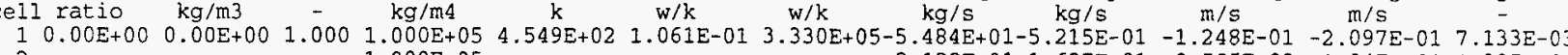

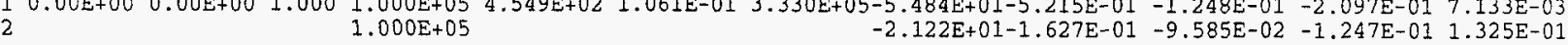

pipe component total coolant mass is $4.44809 \mathrm{E}+02 \mathrm{~kg}$, total coolant energy is $3.46305 \mathrm{E}+08 \mathrm{w} * \mathrm{~s}$, and

computed initial total coolant mass is $4.44938 \mathrm{E}+02 \mathrm{~kg}$

rac large edit

roblem time is $1.102656 \mathrm{E}-02 \mathrm{~s}$, time-step size is $1.4775 \mathrm{E}-03 \mathrm{~s}$, time-step number is 0.00955

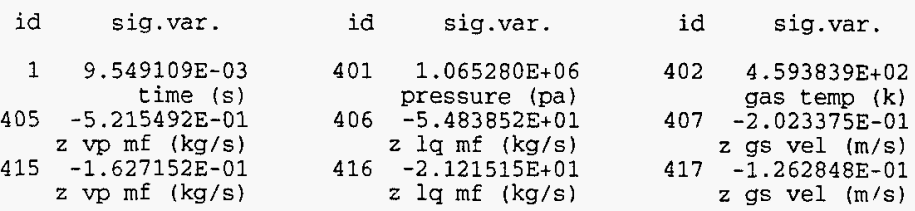

\begin{tabular}{|c|c|}
\hline id & sig.var. \\
\hline 40 & $548663 \mathrm{E}+02$ \\
\hline & $z$ z 1 vel $(\mathrm{m} / \mathrm{s})$ \\
\hline 41 & $\begin{array}{l}-9.591856 \mathrm{E}-0 \\
z \text { lq vel }(\mathrm{m} / \mathrm{s}\end{array}$ \\
\hline
\end{tabular}

id sig.var.

$404 \quad 5.004752 \mathrm{E}-01$ 409 void fraction $2 \mathrm{~m} / \mathrm{mLw}(\mathrm{kg} / \mathrm{s})$ $419-2.137786 \mathrm{E}+01$

the component type is a pipe, component number is 4 , first junction number is 4 , and second junction number is 8 pressure pressure void fr.temp.sat. temp.liq. temp.gas den.liq. den.vap. vel.liq. vel.gas wf.liq.

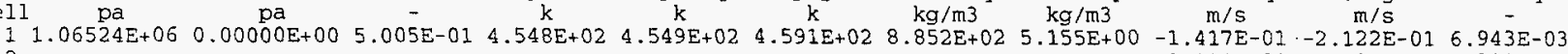

dis.sol. p.o.sol. fr.new if.drag temp.ss. evap. cond. mfw.liq. mfw.vap. velt.liq velt.gas wf.gas

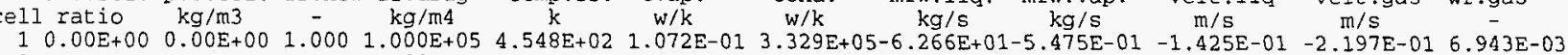
$2 \quad 1.000 \mathrm{E}+05 \quad-2.458 \mathrm{E}+01-1.850 \mathrm{E}-01-1.110 \mathrm{E}-01-1.418 \mathrm{E}-01 \quad 1.323 \mathrm{E}-01$

pipe component total coolant mass is $4.44761 \mathrm{E}+02 \mathrm{~kg}$, total coolant energy is $3.46267 \mathrm{E}+08 \mathrm{~W}^{*} \mathrm{~S}$, and computed initial total coolant mass is $4.44938 \mathrm{E}+02 \mathrm{~kg}$

large edit

problem time is $1.257789 \mathrm{E}-02 \mathrm{a}$, time-step size is $1.5513 \mathrm{E}-03 \mathrm{~s}$, time-step number is 10 , outer-iteration number is 2

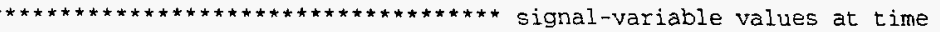

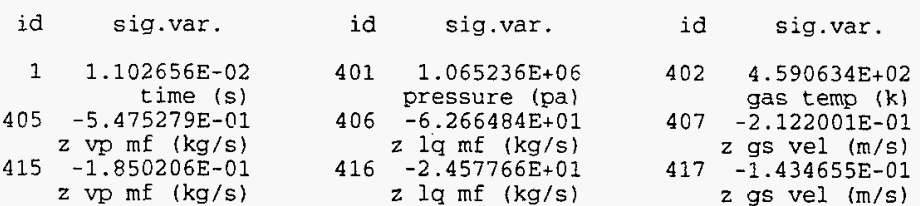

$0.01103 \mathrm{~s}$

the component type is a pipe, component number is 4 , first junction number is 4 , and second junction number is 8

cell pressure pressure void Er. temp.sat. temp.liq. temp.gas den.liq. den.vap. vel.liq. vel.gas wf.liq.

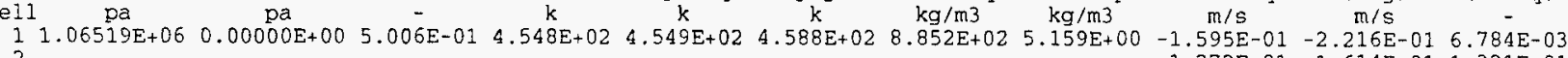
$\begin{array}{rrrr}2 & \end{array}$

dis.sol. p.0.sol. fr.new if.drag temp.ss. evap. cond. mfw.liq. mfw.vap. velt.liq velt.gas wf.gas

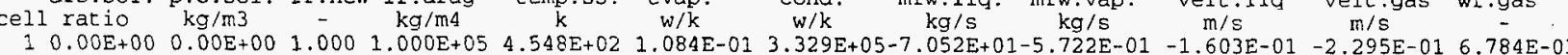

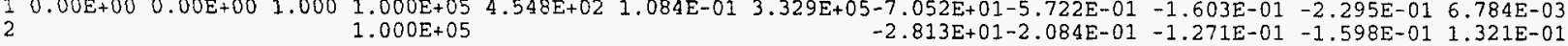

pipe component total coolant mass is $4.44704 \mathrm{E}+02 \mathrm{~kg}$, total coolant energy is $3.46222 \mathrm{E}+08 \mathrm{w}^{\star} \mathrm{S}$, and computed initial total coolant mass is $4.44938 \mathrm{E}+02 \mathrm{~kg}$ 
problem time is $1.420679 \mathrm{E}-02 \mathrm{~s}$, time-step size is $1.6289 \mathrm{E}-03 \mathrm{~s}$, time-step number is 11 , outer-iteration number is 2

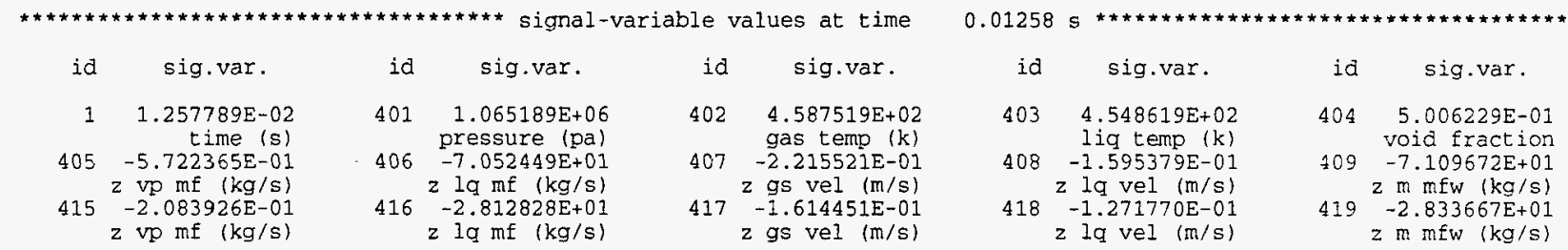

the component type is a pipe, component number is 4 , first junction number is 4 , and second junction number is 8

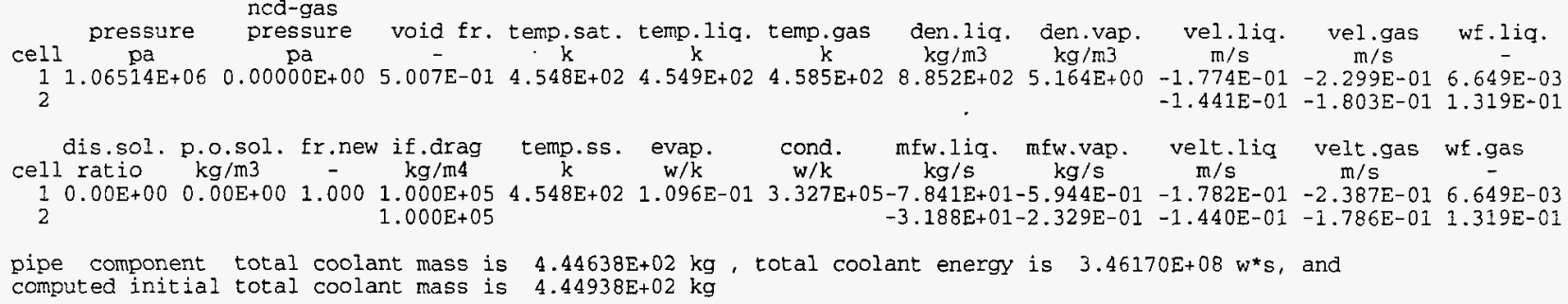

id sig.var.

$1 \quad 1.420679 \mathrm{E}-02$ time (s)

$405-5.944209 \mathrm{E}-01$

$\mathrm{z} \mathrm{vp} \mathrm{mf}(\mathrm{kg} / \mathrm{s})$

$415-2.328753 \mathrm{E}-01$

$\mathrm{z} v \mathrm{pp}(\mathrm{kg} / \mathrm{s})$ id sig.var.

$402 \quad 4.584506 \mathrm{E}+02$

$407 \quad-2.299100 \mathrm{E}-01$

gs vel (m/s)

$417-1.802572 \mathrm{E}-01$ $\mathrm{z}$ gs vel (m/s)
$0.01421 \mathrm{~s}$

id sig.var.

id sig.var.

$403 \quad 4.548596 \mathrm{E}+02$ liq temp (k) $=1 \mathrm{q}$ vel $(\mathrm{m} / \mathrm{s})$

$418-1.441319 \mathrm{E}-01$ $\mathrm{z}$ lq vel (m/s)
$404 \quad 5.007135 E-01$ 409 void fraction $\mathrm{z} \mathrm{mfw}(\mathrm{kg} / \mathrm{s})$

$419-3.211073 \mathrm{E}+01$ $z \mathrm{~m} \mathrm{mfw}(\mathrm{kg} / \mathrm{s})$

the component type is a pipe, component number is 4 , first junction number is 4 , and second -junction number is 8 pressure pressure void fr. temp.sat. temp.liq. temp.gas den.liq. den.vap. vel.liq. vel.gas wf.liq.

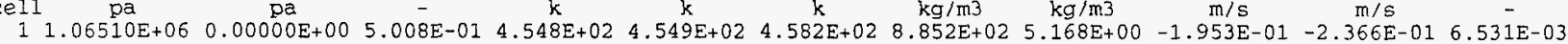
$21.06510 \mathrm{E}+06 \quad 0.00000 \mathrm{E}+00 \quad 5.008 \mathrm{E}-014.548 \mathrm{E}+02 \quad 4.549 \mathrm{E}+02 \quad 4.582 \mathrm{E}+02 \quad 8.852 \mathrm{E}+02 \quad 5.168 \mathrm{E}+00-1.650 \mathrm{E}-01-1.999 \mathrm{E}-01 \quad 1.318 \mathrm{E}-01$

dis.sol. p.0.sol. fr.new if.drag temp.ss. evap. cond. mfw.liq. mfw.vap. velt.liq velt.gas wf.gas

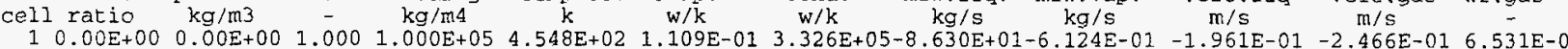

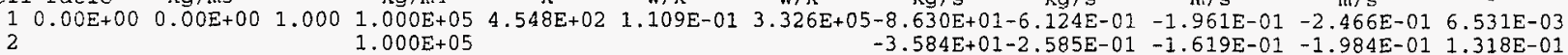

pipe component total coolant mass is $4.44561 \mathrm{E}+02 \mathrm{~kg}$, total coolant energy is $3.46110 \mathrm{E}+08 \mathrm{w}^{*} \mathrm{~s}$, and computed initial total coolant mass is $4.44938 \mathrm{E}+02 \mathrm{~kg}$

problem time is $3.306595 \mathrm{E}-02 \mathrm{~s}$, time-step size is $2.5270 \mathrm{E}-03 \mathrm{~s}$, time-step number is

20, outer-iteration number is 2

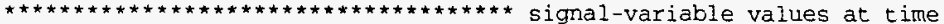

\begin{tabular}{|c|c|c|c|c|}
\hline sig.var. & id & sig.var. & id & sig.var. \\
\hline $\begin{array}{r}3.053900 \mathrm{E}-02 \\
\text { time (s) }\end{array}$ & & $\begin{array}{l}1.064787 E+06 \\
\text { pressure (pa) }\end{array}$ & 402 & $\begin{array}{l}4.564773 \mathrm{E}+02 \\
\text { gas temp }(\mathrm{k})\end{array}$ \\
\hline $05 \begin{array}{r}-6.808401 \mathrm{E}-01 \\
\mathrm{z} \operatorname{vp} \mathrm{mf}(\mathrm{kg} / \mathrm{s})\end{array}$ & & $-1.432853 \mathrm{E}+02$ & 407 & $-2.612941 \mathrm{E}-01$ \\
\hline 5230E-01 & 416 & $33894 \mathrm{E}+01$ & 417 & $-3.651855 E-0$ \\
\hline
\end{tabular}
$0.03054 \mathrm{~s}$

id sig.var.

the component type is a pipe, component number is 4 , first junction number is 4 , and second junction number is 8

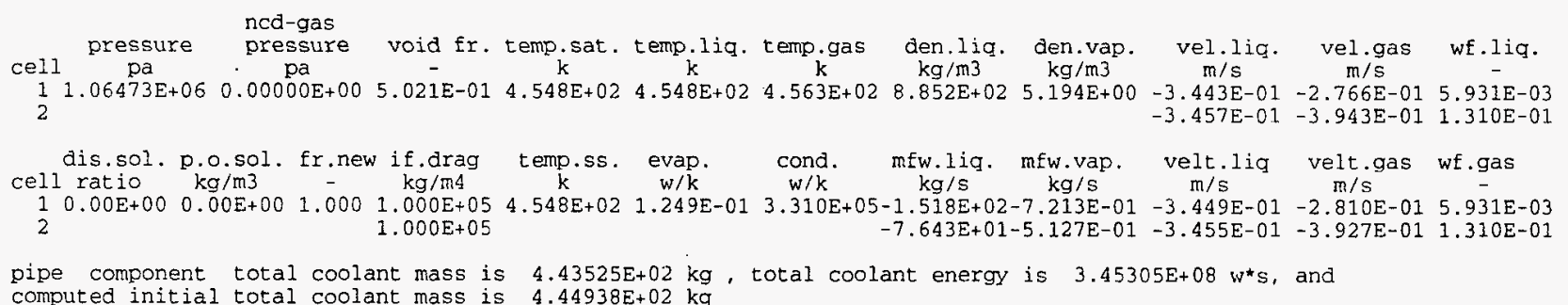


trac large edit

problem time is $3.571925 \mathrm{E}-02 \mathrm{~s}$, time-step size is $2.6533 \mathrm{E}-03 \mathrm{~s}$, time-step number is 21 , outer-iteration number is 2

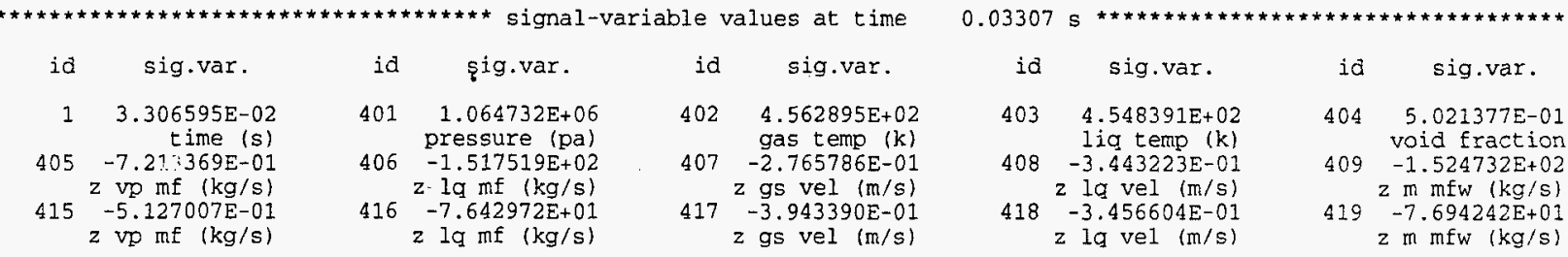

the component type is a pipe, component number is 4 , first junction number is 4 , and second junction number is 8

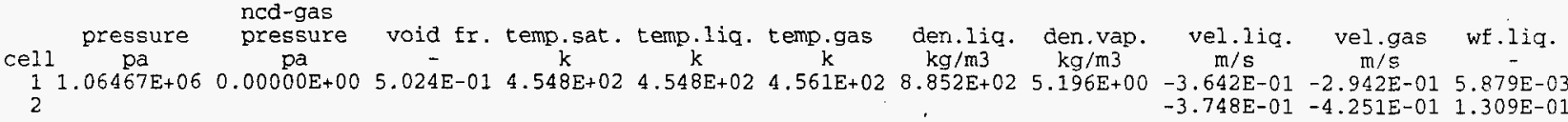

dis.sol. p.o.sol. fr.new if.drag temp.ss. evap. cond. mfw.liq. mfw.vap. velt.liq velt.gas wf.gas

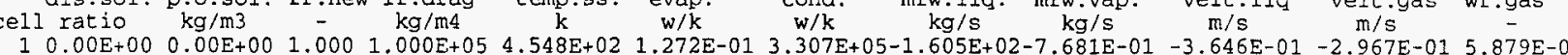

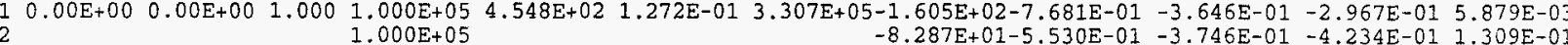
pipe component total coolant mass is $4.43334 \mathrm{E}+02 \mathrm{~kg}$, total coolant energy is $3.45157 \mathrm{E}+08 \mathrm{w}^{\star} \mathrm{s}$, and computed initial total coolant mass is $4.44938 \mathrm{E}+02 \mathrm{~kg}$

trac large edit

problem time is $\mathbf{3 . 8 5 0 5 2 1 E - 0 2} \mathbf{s}$, time-step size is $2.7860 \mathrm{E}-03 \mathrm{~s}$, time-step number is $\mathbf{2 2}$, outer-iteration number is 2

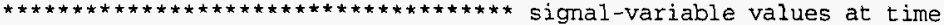

$$
\begin{array}{cc}
\text { id } & \text { sig.var. } \\
1 & 3.571925 \mathrm{E}-02 \\
\mathrm{t} \text { ime (s) } \\
405 \quad-7.680551 \mathrm{E}-01 \\
\mathrm{z} \mathrm{vp}(\mathrm{mg} / \mathrm{s}) \\
415 \quad-5.530478 \mathrm{E}-01 \\
\mathrm{z} \text { vp } \mathrm{mf}(\mathrm{kg} / \mathrm{s})
\end{array}
$$

$\begin{array}{cc}\text { id } & \text { sig.var. } \\ 402 & 4.561152 \mathrm{E}+02 \\ & \text { gas temp (k) } \\ 407 & -2.942200 \mathrm{E}-01 \\ \text { z gs vel (m/s) } \\ 417 \text {-4.251396E-01 } \\ \text { z gs vel (m/s) }\end{array}$
03572

id sig.var.

$403 \quad 4.548367 E+02$ liq temp $(k)$ $408 \quad-3.642474 \mathrm{E}-01$ 2 lq $v e l(m / s)$ $z$ lq vel $(\mathrm{m} / \mathrm{s})$ id sig.var.

$404 \quad 5.023751 E-01$ void fraction $409-1.612253 \mathrm{E}+02$ $2 \mathrm{~m} \mathrm{mfw}(\mathrm{kg} / \mathrm{s})$ $z \mathrm{~m} \mathrm{mfw}(\mathrm{kg} / \mathrm{s})$

the component type is a pipe, component number is 4 , first junction number is 4 , and second junction number is 8

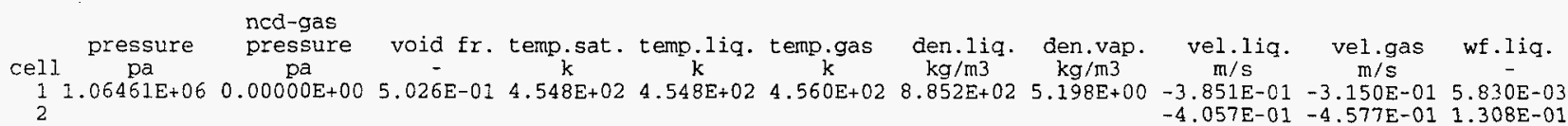

dis.sol. p.0.sol. fr.new if.drag temp.ss, evap. cond. mfw.liq. mfw.vap. velt.liq velt.gas wf.gas $\begin{array}{ccccccccccc}1 & & & \end{array}$

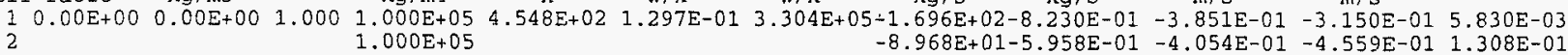
pipe component total coolant mass is $4.43128 \mathrm{E}+02 \mathrm{~kg}$, total coolant energy is $3.44997 \mathrm{E}+08 \mathrm{w}^{\star} \mathrm{s}$, and computed initial total coolant mass is $4.44938 \mathrm{E}+02 \mathrm{~kg}$

trac large edit

problem time is $\mathbf{4 . 1 4 3 0 4 8 \mathrm { E } - 0 2} \mathbf{8}$, time-step size is $2.9253 \mathrm{E}-03 \mathrm{~s}$, time-step number is 23 , outer-iteration number is 2

$13.850521 \mathrm{E}-02$ time (s)

$405-8.230028 E-01$ $2 \mathrm{vp} \mathrm{mf}(\mathrm{kg} / \mathrm{s})$

$415-5.957680 \mathrm{E}-01$ $z$ vp $\mathrm{me}(\mathrm{kg} / \mathrm{s})$ id sig.var.

$\begin{array}{cc}\text { id } & \text { sig.var. } \\ 401 & 1.064611 \mathrm{E}+06 \\ & \text { pressure (pa) } \\ 406 \quad-1.695725 \mathrm{E}+02 \\ \text { z lq mf (kg/s) } \\ 416 \quad-8.96824 \mathrm{E}+01 \\ \text { z lq mf (kg/s) }\end{array}$

the component type is a pipe, component number is

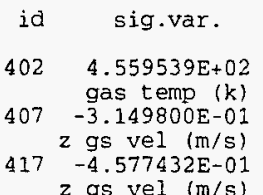

$0.03851 \mathrm{~s} * * *$

\section{id sig.var.}

$4034.548341 \mathrm{E}+02$

$408 \quad$ liq temp (k) 2 iq vel (m/s)

$418-4.056514 \mathrm{E}-01$ $\mathrm{z}$ lq vel (m/s) id sig.var.

$404 \quad 5.026315 E-01$ 409 void fraction

$419 \begin{aligned} & z \mathrm{mfw}(\mathrm{kg} / \mathrm{s}) \\ & -9.027818 \mathrm{E}+01\end{aligned}$ $\mathrm{z} \mathrm{m} \mathrm{mfw}(\mathrm{kg} / \mathrm{s})$

4, first junction number is 4 , and second junction number is 8 cell pressure pressure void fr.temp.sat. temp.liq. temp.gas den.liq. den.vap. vel.liq. vel.gas wf.liq.

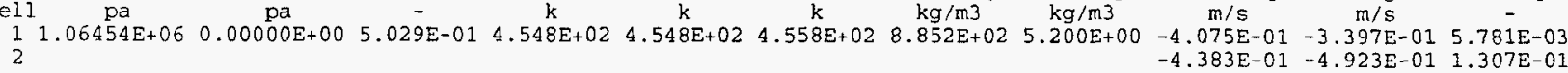
dis.sol. p.o.sol. fr.new if.drag temp.ss. evap. cond. mfw.liq. mfw.vap. velt.liq velt.gas wf.gas

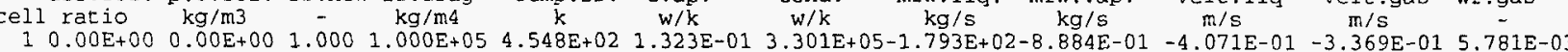

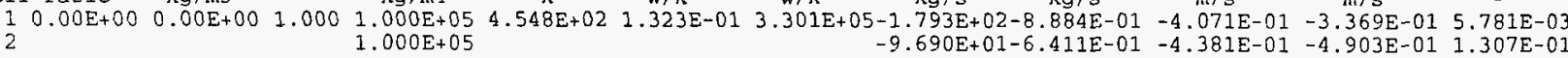
pipe component total coolant mass is $4.42905 \mathrm{E}+02 \mathrm{~kg}$, total coolant energy is $3.44824 \mathrm{E}+08 \mathrm{~W}^{\star} \mathrm{S}$, and computed initial total coolant mass is $4.44938 \mathrm{E}+02 \mathrm{~kg}$ 
trac large edit

problem time is 4.450200E-02 8 , time-step size is $3.0715 \mathrm{E}-03 \mathrm{~s}$, time-step number is $\mathbf{2 4}$, outer-iteration number is 2

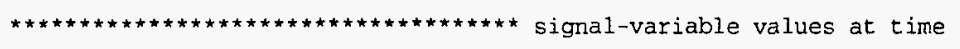

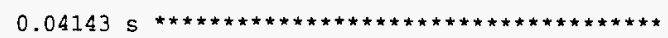

\begin{tabular}{|c|c|c|}
\hline sig.var. & id & sig.var. \\
\hline $\begin{array}{c}4.143048 \mathrm{E}-02 \\
\text { time (s) }\end{array}$ & 401 & $\begin{array}{l}1.064540 \mathrm{E}+06 \\
\text { pressure (pa) }\end{array}$ \\
\hline$-8.54351 \mathrm{E}-01$ & 406 & $32245+02$ \\
\hline $\begin{array}{l}\mathrm{z} \operatorname{vpmf}(\mathrm{kg} / \mathrm{s}) \\
-6.411084 \mathrm{E}-01 \\
z \operatorname{vpmf}(\mathrm{kg} / \mathrm{s})\end{array}$ & 416 & $\begin{array}{l}\mathrm{z} \text { Iq } \mathrm{mf}(\mathrm{kg} / \mathrm{s}) \\
-9.689693 \mathrm{E}+01 \\
\mathrm{z} \text { lg } \mathrm{mf}(\mathrm{kg} / \mathrm{s})\end{array}$ \\
\hline
\end{tabular}

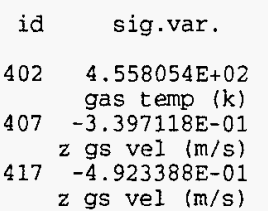

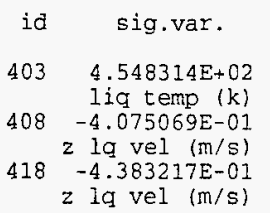

id sig.var.

$4045.029090 \mathrm{E}-01$ 409 void fraction $409-1.802109 \mathrm{E}+02$ $419 \begin{array}{r}z \mathrm{~m} \mathrm{mF} \\ -9.753803 \mathrm{E}+01\end{array}$ $\mathrm{z} \mathrm{m} \mathrm{mfw}(\mathrm{kg} / \mathrm{s})$

the component type is a pipe, component number is 4 , first junction number is 4 , and second junction number is 8

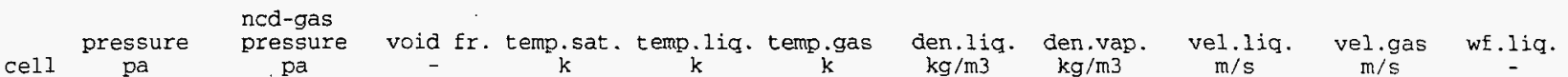

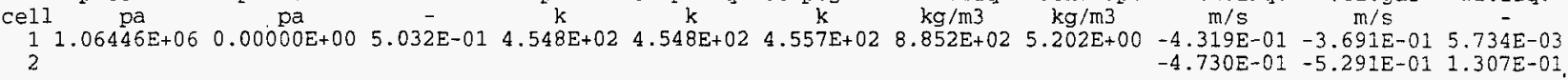

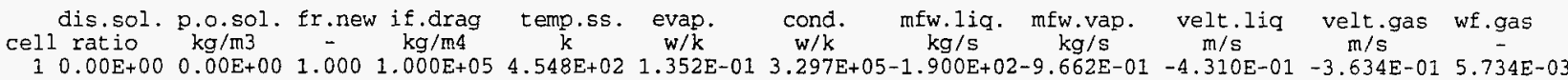

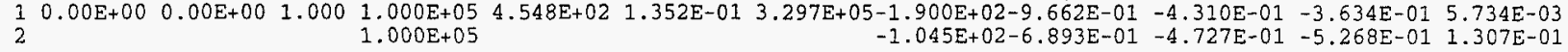
pipe component total coolant mass is $4.42663 \mathrm{E}+02 \mathrm{~kg}$, total coolant energy is $3.44637 \mathrm{E}+08 \mathrm{w}^{*} \mathrm{~s}$, and computed initial total coolant mass is $4.44938 \mathrm{E}+02 \mathrm{~kg}$

trac large edit

problem time is $\mathbf{4 7 7 2 7 1 0 \mathrm { E } - 0 2} \mathrm{g}$, time-step size is $3.2251 \mathrm{E}-03 \mathrm{~s}$, time-step number is 25, outer-iteration number is 2

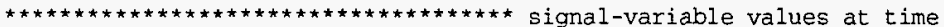
$0.04450 \mathrm{~s}$

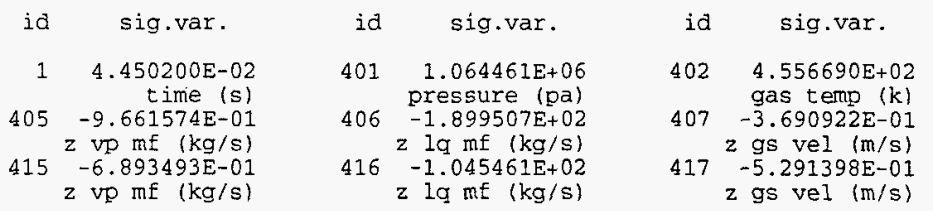

\begin{tabular}{|c|c|}
\hline id & sig.var. \\
\hline 40 & $\begin{array}{l}6 \mathrm{E}+02 \\
0(k)\end{array}$ \\
\hline 0 & $\begin{array}{l}-4.319201 \mathrm{E}-01 \\
\mathrm{z} \text { lg vel }(\mathrm{m} / \mathrm{s})\end{array}$ \\
\hline & $\begin{array}{l}-4.729714 \mathrm{E}-01 \\
=\mathrm{lq} \text { vel }(\mathrm{m} / \mathrm{s})\end{array}$ \\
\hline
\end{tabular}

id sig.var
$404 \quad 5.032107 \mathrm{E}-01$
$\begin{array}{r}\text { void fraction } \\ 409 \quad-1.909168 \mathrm{E}+02 \\ \mathrm{zm} \mathrm{mfw}(\mathrm{kg} / \mathrm{s})\end{array}$
$\begin{aligned}-1.052354 \mathrm{E}+02 \\ \mathrm{zm} \mathrm{mfw}(\mathrm{kg} / \mathrm{s})\end{aligned}$

the compnnent type is a pipe, component number is 4 , first junction number is 4 , and second junction number is 8

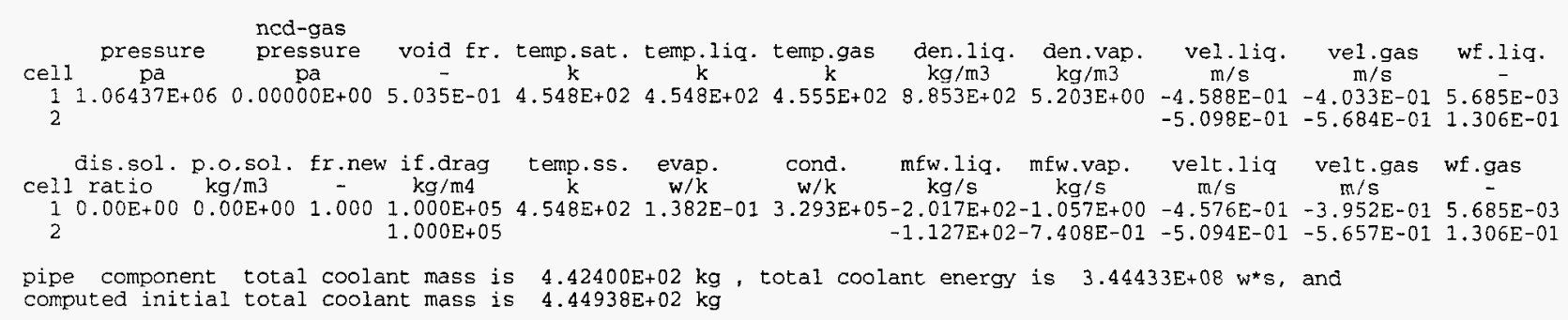

trac large edit

problem time is $9.023112 \mathrm{E}+03 \mathrm{~s}$, time-step size is $1.0015 \mathrm{E}+02 \mathrm{~s}$, time-step number is 315 , outer-iteration number is 1

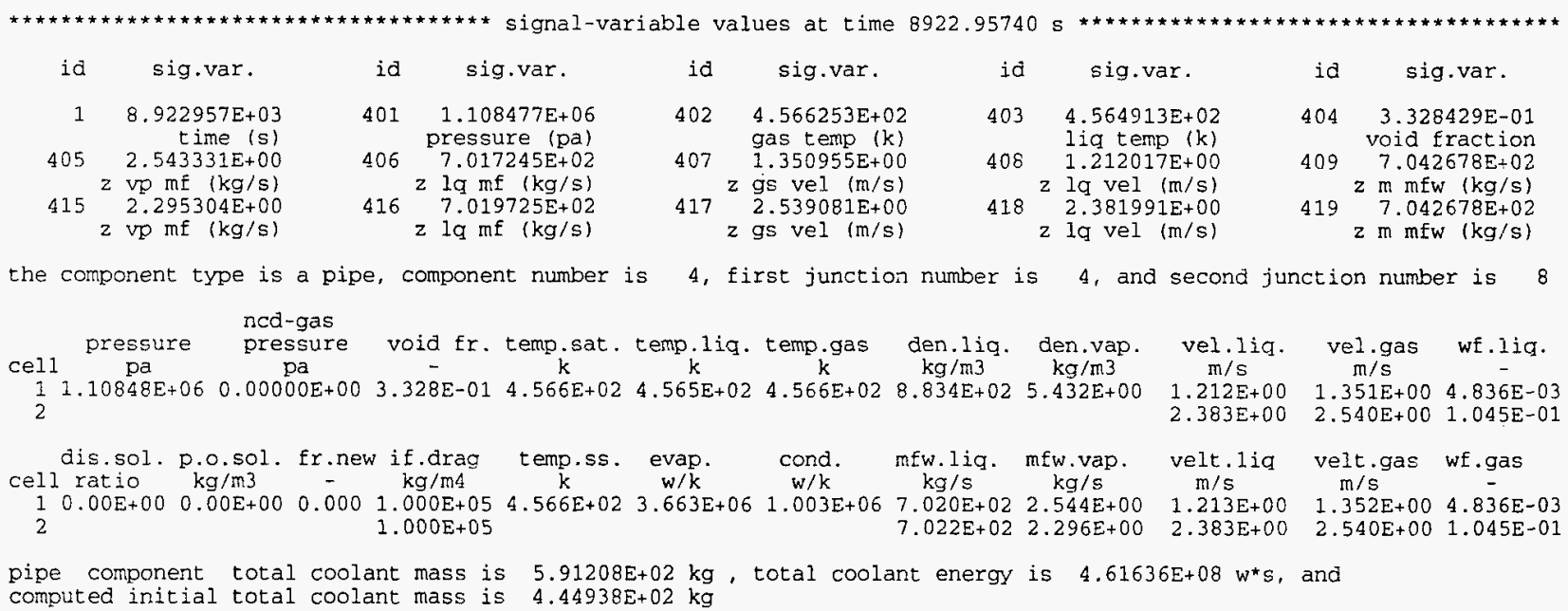


trac large edit

problem time is $9.123267 \mathrm{E}+03 \mathrm{~s}$, time-step size is $1.0015 \mathrm{E}+02 \mathrm{~s}$, time-step number is 316 , outer-iteration number is 1

\begin{tabular}{|c|c|c|c|c|c|c|c|c|c|}
\hline id & sig.var. & id & sig.var. & id & sig.var. & $i d$ & sig.var. & id & sig.var. \\
\hline & $\begin{array}{r}9.023112 \mathrm{E}+03 \\
\text { time (s) }\end{array}$ & & $\begin{array}{r}1.108477 E+06 \\
\text { pressure (pa) }\end{array}$ & 402 & $\begin{array}{l}4.566253 E+02 \\
\text { gas temp (k) }\end{array}$ & 403 & $\begin{array}{l}4.564913 E+02 \\
\text { liq temp (k) }\end{array}$ & & $\begin{array}{r}3.328388 \mathrm{E}-01 \\
\text { void fraction }\end{array}$ \\
\hline 405 & $2.544103 E+00$ & 406 & $7.019 \cdot: 6 \mathrm{E}+02$ & 407 & $1.351365 E+0 r$ & 408 & $1.212427 E+00$ & 409 & $7.045057 \mathrm{E}+02$ \\
\hline $415^{2}$ & $\begin{array}{l}\mathrm{vp} \mathrm{mf}(\mathrm{kg} / \mathrm{s}) \\
2.295992 \mathrm{E}+00 \\
\mathrm{vp} \mathrm{mE}(\mathrm{kg} / \mathrm{s})\end{array}$ & 416 & $\begin{array}{l}\text { z lq } \mathrm{mf}(\mathrm{kg} / \mathrm{s}) \\
7.022097 \mathrm{E}+02 \\
z \mathrm{lg} \mathrm{mf}(\mathrm{kg} / \mathrm{s})\end{array}$ & 417 & $\begin{array}{l}\text { gs vel }(\mathrm{m} / \mathrm{s}) \\
2.539872 \mathrm{E}+00 \\
\text { gs vel (m/s) }\end{array}$ & 418 & $\begin{array}{l}\text { lq vel (m/s) } \\
2.382782 \mathrm{E}+00 \\
\text { lq vel (m/s) }\end{array}$ & 419 & $\begin{array}{l}\mathrm{zmmw}(\mathrm{kg} / \mathrm{s}) \\
7.045057 \mathrm{E}+02 \\
\mathrm{zmmf}(\mathrm{kg} / \mathrm{s})\end{array}$ \\
\hline
\end{tabular}

the component type is a pipe, component number is 4 , first junction number is 4 , and second junction number is 8

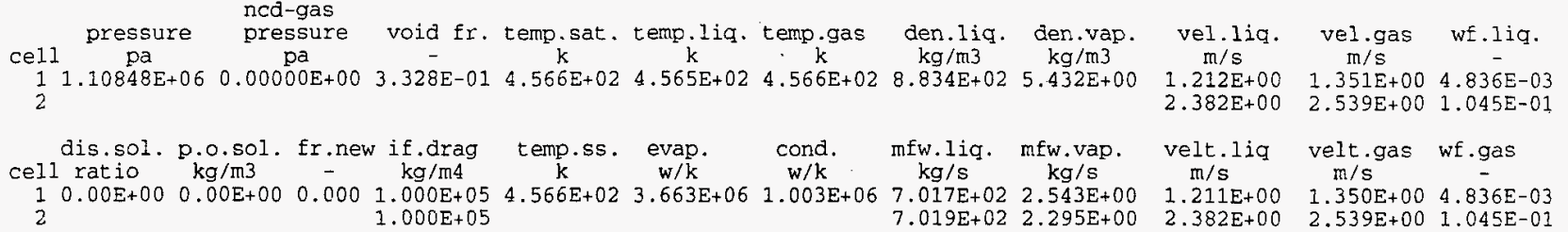
pipe component total coolant mass is $5.91211 \mathrm{E}+02 \mathrm{~kg}$, total coolant energy is $4.61639 \mathrm{E}+08 \mathrm{w}^{*} \mathrm{~s}$, and computed initial total coolant mass is $4.44938 \mathrm{E}+02 \mathrm{~kg}$

trac large edit

problem time is $\mathbf{9 . 2 2 3 4 2 2 E + 0 3} \mathbf{8}$, time-step size is $1.0015 E+02 \mathrm{~s}$, time-step number is 317 , outer-iteration number is 1

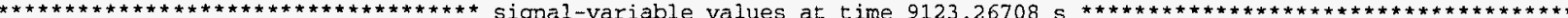

\begin{tabular}{|c|c|c|c|c|c|c|c|c|c|}
\hline id & sig.var. & id & sig.var. & id & sig.var. & id & sig.var. & id & sig.var. \\
\hline 1 & $\begin{array}{r}9.123267 \mathrm{E}+03 \\
\text { time (s) }\end{array}$ & 401 & $\begin{array}{r}1.108477 E+06 \\
\text { pressure (pa) }\end{array}$ & 402 & $\begin{array}{l}4.566253 \mathrm{E}+02 \\
\text { gas temp }(\mathrm{k})\end{array}$ & 403 & $\begin{array}{l}4.564913 \mathrm{E}+02 \\
\text { lig temp (k) }\end{array}$ & 404 & $\begin{array}{l}3.328440 E- \\
\text { void fractic }\end{array}$ \\
\hline 5 & $2.543116 E+00$ & 406 & $7.016583 \mathrm{E}+02$ & 407 & $1.350841 \mathrm{E}+00$ & 408 & $1.211903 E+00$ & 409 & \\
\hline 5 & $\begin{array}{l}\mathrm{vp} \operatorname{mf}(\mathrm{kg} / \mathrm{s}) \\
2.295112 \mathrm{E}+00 \\
\mathrm{vp} \mathrm{mf}(\mathrm{kg} / \mathrm{s})\end{array}$ & 416 & $\begin{array}{l}\text { z } 1 \mathrm{mf}(\mathrm{kg} / \mathrm{s}) \\
7.019064 \mathrm{~s}+02 \\
\mathrm{z} \operatorname{lq} \mathrm{mf}(\mathrm{kg} / \mathrm{s})\end{array}$ & $417^{2}$ & $\begin{array}{l}\text { gs vel (m/s) } \\
2.538861 \mathrm{E}+00 \\
\text { gs vel (m/s) }\end{array}$ & 418 & $\begin{array}{l}1 q \text { vel }(\mathrm{m} / \mathrm{s}) \\
2.381771 \mathrm{E}+00 \\
\text { lq vel }(\mathrm{m} / \mathrm{s})\end{array}$ & 419 & $\begin{array}{l}\text { z m mfw }(\mathrm{kg} / \mathrm{s} \\
7.042015 \mathrm{E}+0 \\
\mathrm{z} \mathrm{m} \mathrm{mEw}(\mathrm{kg} / \mathrm{s}\end{array}$ \\
\hline
\end{tabular}

the component type is a pipe, component number is 4 , first junction number is 4 , and second junction number is 8

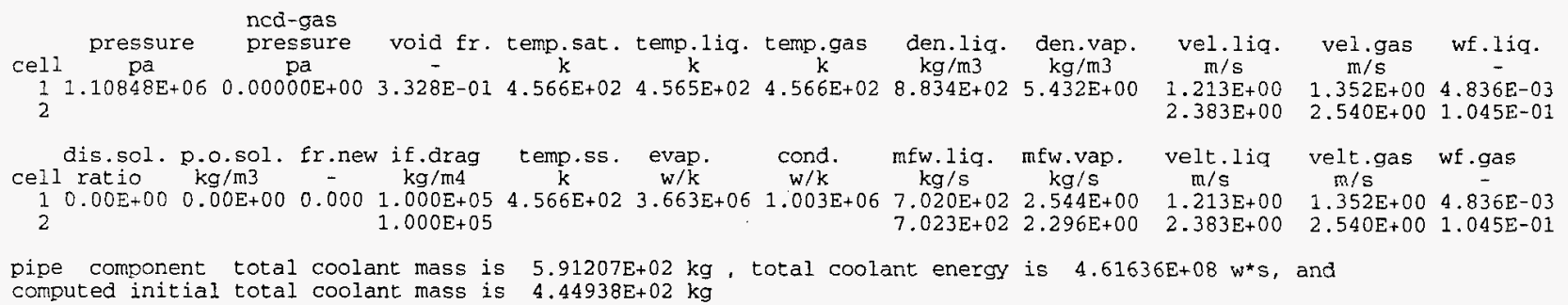
trac large edit

problem time is $9.323577 \mathrm{E}+03 \mathrm{~s}$, time-step size is $1.0015 \mathrm{E}+02 \mathrm{~s}$, time-step number is 318 , outer-iteration number is 1

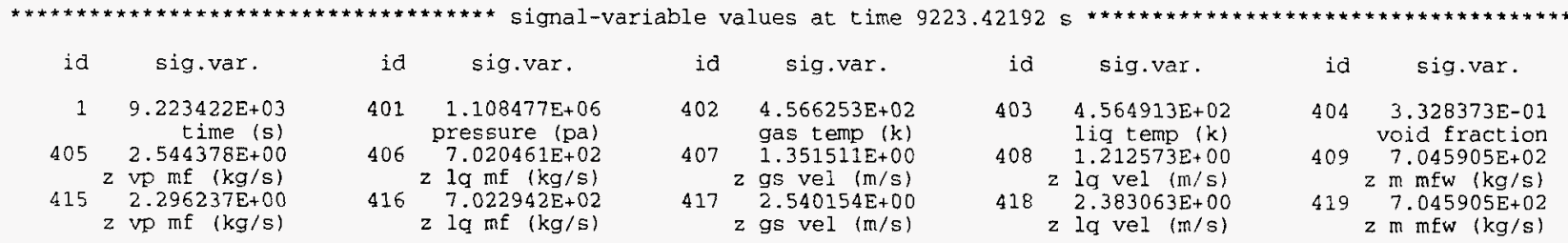
the component type is a pipe, component number is 4 , first junction number is 4 , and second junction number is 8 pressure pressure void fr. temp.sat. temp.liq. temp.gas den.liq. den.vap. vel.liq. vel.gas wf.liq.

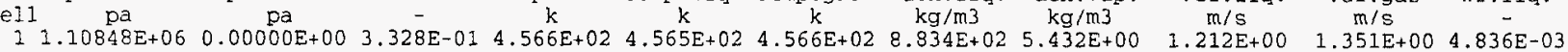
2

dis.sol. p.o.sol. fr.new if.drag temp.ss, evap. cond. mfw.lig. mfw.vap. velt.liq velt.gas wf.gas cell ratio $\mathrm{kg} / \mathrm{m3} \quad-\mathrm{kg} / \mathrm{m} 4 \mathrm{w} / \mathrm{k} / \mathrm{k} / \mathrm{k} \mathrm{kg} / \mathrm{s}$ kg/s $\mathrm{m} / \mathrm{s} / \mathrm{s}$

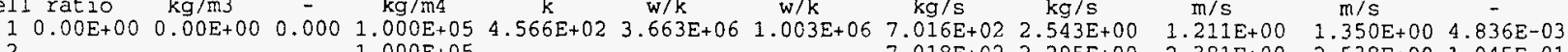

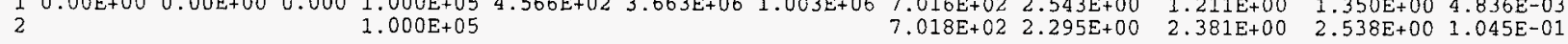
pipe component total coolant mass is $5.91213 \mathrm{E}+02 \mathrm{~kg}$, total coolant energy is $4.61640 \mathrm{E}+08 \mathrm{w}^{\star} \mathrm{s}$, and computed initial total coolant mass is $4.44938 \mathrm{E}+02 \mathrm{~kg}$ 
26641 trac large edit

problem time is $9.423732 \mathrm{E}+03 \mathrm{~s}$, time-step size is $1.0015 \mathrm{E}+02 \mathrm{~s}$, time-step number is 319 , outer-iteration number is 1

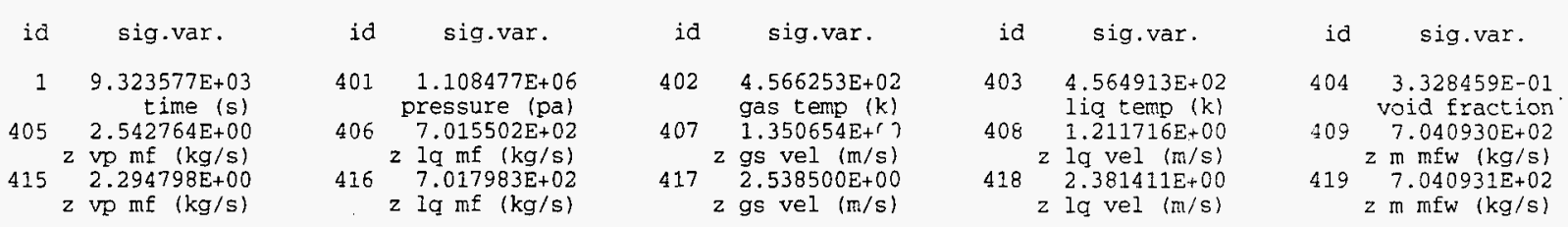

the component type is a pipe, component number is 4 , first junction number is 4 , and second junction number is 8

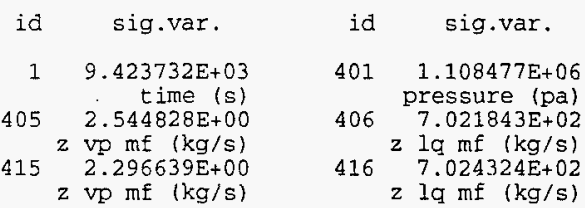

the component type is a pipe, component number is

id sig.var.

$402 \quad 4.566253 E+02$

$407 \quad$ gas temp $(\mathrm{k})$ $\mathrm{z}$ gs vel $(\mathrm{m} / \mathrm{s})$

$417 \quad 2.540615 \mathrm{E}+00$ $z$ gs vel $(\mathrm{m} / \mathrm{s})$

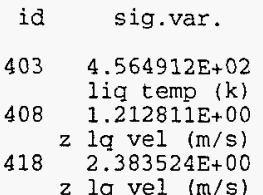

id sig.var

$404 \quad 3.328349 E-01$ void fraction $409 \quad 7.047292 \mathrm{E}+02$ $419^{2} \mathrm{~m} \mathrm{mfw}(\mathrm{kg} / \mathrm{s})$ $2 \mathrm{~m} \mathrm{mfw}(\mathrm{kg} / \mathrm{s})$

\section{pressure pressure void fr. temp.sat. temp.liq. temp.gas den.liq. den.vap. vel.liq. vel.gas wf.liq.

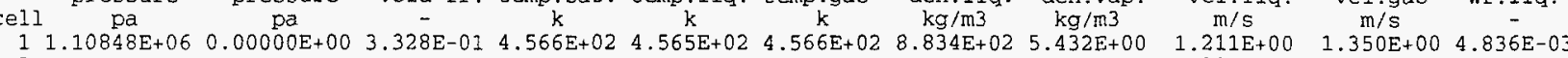 $\begin{array}{llll}1 & \\ 2.381 \mathrm{E}+00 & 2.538 \mathrm{E}+00 & 1.045 \mathrm{E}-01\end{array}$ \\ dis.sol. p.0.sol. Er.new if.drag temp.ss. evap. cond. mfw.liq. mfw.vap. velt.liq velt.gas wf.gas

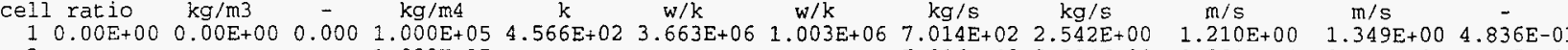

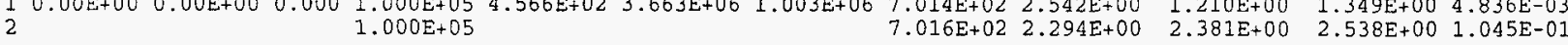 \\ pipe component total coolant mass is $5.91215 \mathrm{E}+02 \mathrm{~kg}$, total coolant energy is $4.61642 \mathrm{E}+08 \mathrm{w}^{*} \mathrm{~s}$, and computed initial total coolant mass is $4.44938 \mathrm{E}+02 \mathrm{~kg}$}

problem time is $9.624041 \mathrm{E}+03 \mathrm{~s}$, time-step size is $1.0015 \mathrm{E}+02 \mathrm{~s}$, time-step number is $\mathbf{3 2 1}$, outer-iteration number is 1

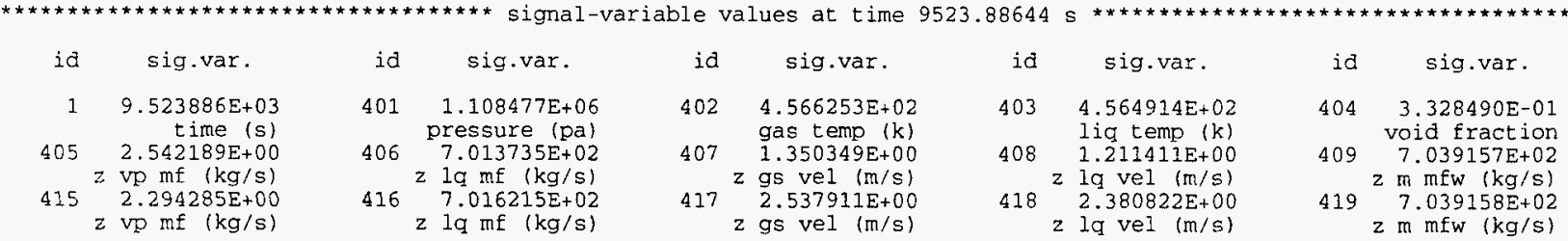
the component type is a pipe, component number is 4 , first junction number is 4 , and second junction number is 8 cell pressure pressure void fr. temp.sat. temp.liq. temp.gas den.liq. den.vap. vel.liq. vel.gas wf.liq.

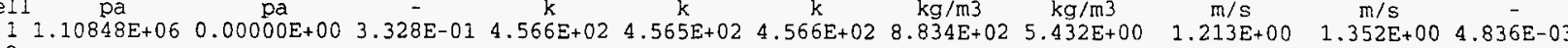

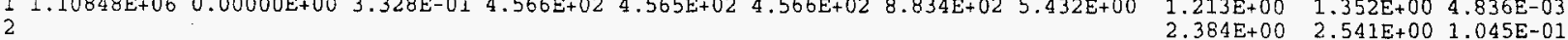
dis.sol. p.0.sol. fr.new if.drag temp.ss. evap. cond. mfw.liq. mfw.vap. velt.liq velt.gas wf.gas

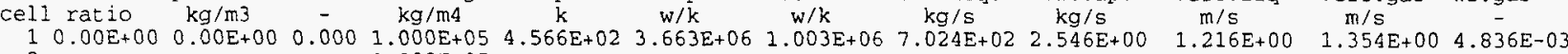

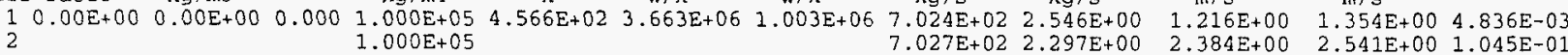
pipe component total coolant mass is $5.91202 \mathrm{E}+02 \mathrm{~kg}$, total coolant energy is $4.61632 \mathrm{E}+08 \mathrm{w}^{\star} \mathrm{s}$, and computed initial total coolant mass is $4.44938 \mathrm{E}+02 \mathrm{~kg}$ 
2912

29130

29131

2915

2915

29156

29157

2915

29160
29161

29162

29163

29196

29197

29198

29199

29200

29201
29202

29203

29204

29205

29206

29207
29208

29208
29209

29210
29211

29957

29958

29959

2996

29983

29984
29985

29986

29987

29988

29989

29990

29991

29992

30025

30026
30027

30028

30030

30031

30032

30033

30034

30035

30036
30037

30038

30039

30040

30786

30787

30788

3078

30812

30813

30814

30815

30816

30817

30818

30819

30820

30821

3085

30855

3085

3085

30858

30859

30860

30861

30862

30863

30864

30865

3086

30867

30868

30869

problem time is $9.724196 \mathrm{E}+03 \mathrm{~s}$, time-step size is $1.0015 \mathrm{E}+02 \mathrm{~s}$, time-step number is $\mathbf{3 2 2}$, outer-iteration number is 1

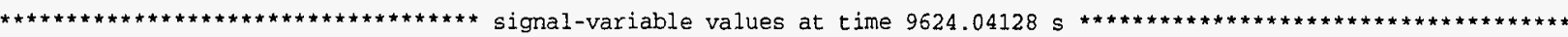

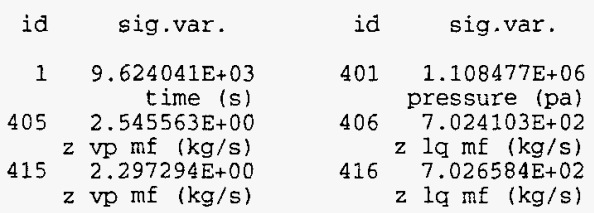

the component type is a pipe, component number is 4 , first junction number is 4 , and second junction number is 8

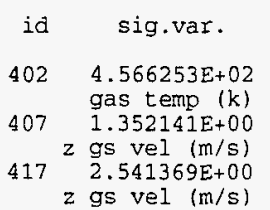

id

sig.var.

\section{$403 \quad 4.564912 \mathrm{E}+02$}

Iiq temp (k)

$408 \cdot 1.213202 \mathrm{E}+00$

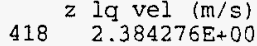

$z$ lq vel $(\mathrm{m} / \mathrm{s})$ id sig.var.

$4043.328310 E-01$

$409 \quad 7.049558 \mathrm{E}+02$

$409 \begin{aligned} & 7.049558 \mathrm{E}+02 \\ & \mathrm{z} \mathrm{m} \mathrm{mfw} \mathrm{(kg/s)}\end{aligned}$

$419^{2} \mathrm{~m}$ mfw $(\mathrm{kg} / \mathrm{s})$

pressure pressure void fr.temp.sat. temp.liq. temp.gas den.liq. den.vap. vel.liq. vel.gas wf.liq.

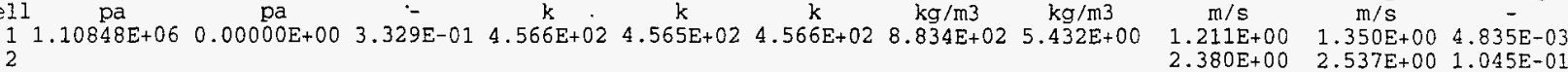

dis.sol. p.o.sol. Er.new if.drag temp.ss. evap. cond. mfw.liq. mfw.vap. velt.lig velt.gas wf.gas

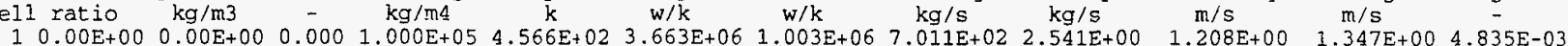

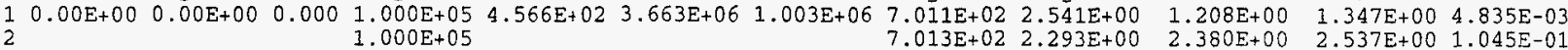

pipe component total coolant mass is $5.91218 \mathrm{E}+02 \mathrm{~kg}$, total coolant energy is $4.61644 \mathrm{E}+08 \mathrm{w}^{*} \mathrm{~s}$, and

computed initial total coolant mass is $4.44938 \mathrm{E}+02 \mathrm{~kg}$

trac large edit

problem time is $9.824351 \mathrm{E}+03 \mathrm{~s}$, time-step size is $1.0015 \mathrm{E}+02 \mathrm{~s}$, time-step number is 323 , outer-iteration number is 1

\begin{tabular}{|c|c|c|c|c|c|c|c|c|c|}
\hline id & sig.var. & id & sig.var. & id & sig.var. & id & sig.var. & id & sig.var. \\
\hline $\begin{array}{r}1 \\
405\end{array}$ & $\begin{array}{r}9.724196 \mathrm{E}+03 \\
\text { time (s) } \\
2.541249 \mathrm{E}+00\end{array}$ & $\begin{array}{l}401 \\
406\end{array}$ & $\begin{array}{c}1.108477 \mathrm{E}+06 \\
\text { pressure }(\mathrm{pa}) \\
7.010844 \mathrm{E}+02\end{array}$ & $\begin{array}{l}402 \\
407\end{array}$ & $\begin{array}{l}4.566253 \mathrm{E}+02 \\
\text { gas temp (k) } \\
1.349849 \mathrm{k}+00\end{array}$ & $\begin{array}{l}403 \\
408\end{array}$ & $\begin{array}{l}4.564914 \mathrm{E}+02 \\
1 \mathrm{ig} \text { temp (k) } \\
1.210912 \mathrm{E}+00\end{array}$ & $\begin{array}{l}404 \\
409\end{array}$ & $\begin{array}{r}3.328540 \mathrm{E}-01 \\
\text { void fraction } \\
7.036257 \mathrm{E}+02\end{array}$ \\
\hline $415^{z}$ & $\begin{array}{l}\mathrm{vp} \mathrm{mf}(\mathrm{kg} / \mathrm{s}) \\
2.293446 \mathrm{E}+00 \\
\mathrm{vp} \mathrm{mf}(\mathrm{kg} / \mathrm{s})\end{array}$ & 416 & $\begin{array}{l}z \operatorname{lq} \mathrm{mf}(\mathrm{kg} / \mathrm{s}) \\
7.013324 \mathrm{E}+02 \\
z \mathrm{lq} \mathrm{mf}(\mathrm{kg} / \mathrm{s})\end{array}$ & 417 & $\begin{array}{l}\text { gs vel (m/s) } \\
2.536947 \mathrm{E}+00 \\
\text { gs vel (m/s) }\end{array}$ & 418 & $\begin{array}{l}\text { lq vel (m/s) } \\
2.379859 \mathrm{E}+00 \\
\text { lq vel }(\mathrm{m} / \mathrm{s})\end{array}$ & 419 & $\begin{array}{l}\mathrm{z} \mathrm{mfw}(\mathrm{kg} / \mathrm{s}) \\
7.036259 \mathrm{E}+02 \\
\mathrm{z} \mathrm{m} \mathrm{mfw}(\mathrm{kg} / \mathrm{s})\end{array}$ \\
\hline
\end{tabular}

the component type is a pipe, component number is 4 , first junction number is 4 , and second junction number is 8

pressure pressure void fr.temp.sat. temp.liq. temp.gas den.liq. den.vap. vel.liq. vel.gas wf.liq.

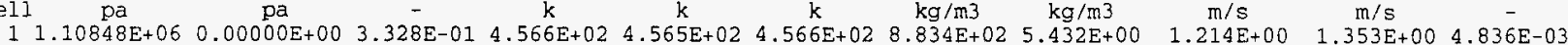

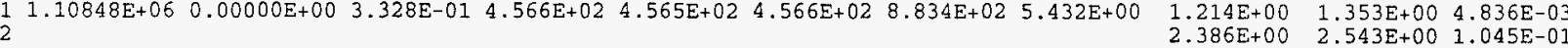

dis.sol. p.o.sol. fr.new if.drag temp.ss. evap. cond. mfw.liq. mfw.vap. velt.liq velt.gas wf.gas $\begin{array}{cccccccccccc} & & & \end{array}$

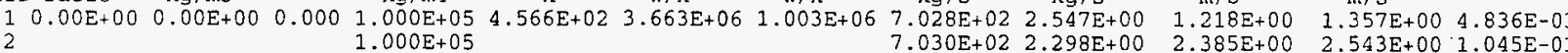

pipe component total coolant mass is $5.91198 \mathrm{E}+02 \mathrm{~kg}$, total coolant energy is $4.61629 \mathrm{E}+08 \mathrm{w*}$, and computed initial total coolant mass is $4.44938 \mathrm{E}+02 \mathrm{~kg}$

rac large edit

problem time is $9.924506 \mathrm{E}+03 \mathrm{~B}$, time-step size is $1.0015 \mathrm{E}+02 \mathrm{~s}$, time-step number is 324 , outer-iteration number is 1

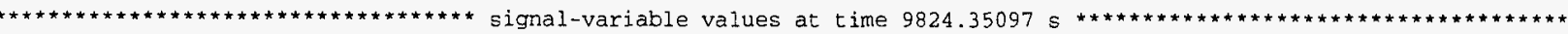

id sig.var.

id sig.var.

$401 \quad 1.108477 \mathrm{E}+06$

$406 \quad 7.027797 \mathrm{E}+02$

$\mathrm{z} \operatorname{lq} \mathrm{mf}(\mathrm{kg} / \mathrm{s})$

$416 \quad 7.030278 \mathrm{E}+02$

$\mathrm{z} \mathrm{lq} \mathrm{mf}(\mathrm{kg} / \mathrm{s})$

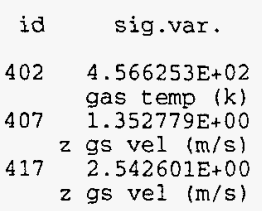

id

sig.var.

$4034.564911 \mathrm{E}+02$

408 liq temp (k)

$408 \quad 1.213840 \mathrm{E}+00$

418 z lq vel (m/s)

$z$ lq vel $(\mathrm{m} / \mathrm{s})$ id sig.var.

$404 \quad 3.328247 \mathrm{E}-01$

void fraction

$0097.053264 \mathrm{E}+02$

$2 \mathrm{~m} \mathrm{mfw}(\mathrm{kg} / \mathrm{s})$

$7.053262 \mathrm{E}+02$
$\mathrm{~m} \mathrm{~m} \mathrm{mfw}(\mathrm{kg} / \mathrm{s})$

the component type is a pipe, component number is 4, first junction number is 4 , and second junction number is 8 pressure pressure void fr.temp.sat. temp.liq. temp.gas den.liq. den.vap. vel.liq. vel.gas wf.liq.

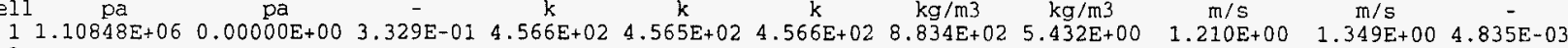

dis.sol. p.o.sol. fr.new if.drag temp.ss. evap. cond. mfw.liq. mfw.vap. velt.liq velt.gas wf.gas $\begin{array}{ccccccccccccc} & & & \end{array}$ $\begin{array}{lllll}1.000 \mathrm{E}+05 & 7.009 \mathrm{E}+02 & 2.292 \mathrm{E}+00 & 2.378 \mathrm{E}+00 & 2.535 \mathrm{E}+00 \quad 1.045 \mathrm{E}-01\end{array}$

pipe component total coolant mass is $5.91224 \mathrm{E}+02 \mathrm{~kg}$, total coolant energy is $4.61649 \mathrm{E}+08 \mathrm{w}^{\star} \mathrm{S}$, and computed initial total coolant mass is $4.44938 \mathrm{E}+02 \mathrm{~kg}$ 
trac large edit

31615

31612

31618

31641

31642

31643

31644

31645

31646

31647

31648

31649

31650

31683

31684

31685

31686

31687

31688

31589

31590

31691

31692

31693

31694

31695

31696

31697

31698

problem time is $1.002466 \mathrm{E}+04 \mathrm{~s}$, time-step size is $1.0015 \mathrm{E}+02 \mathrm{~s}$, time-step number is 325 , outer-iteration number is 1

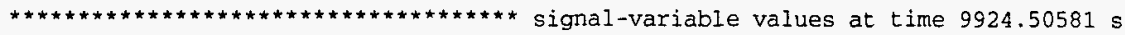

\begin{tabular}{|c|c|c|c|c|c|}
\hline id & var. & id & sig.var. & id & sig.var. \\
\hline 1 & $\begin{array}{c}9.924506 E+03 \\
\text { time (s) }\end{array}$ & 401 & $\begin{array}{r}1.108477 \mathrm{E}+06 \\
\text { pressure (pa) }\end{array}$ & 402 & $\begin{array}{l}4.566253 E+02 \\
\text { gas temp (k) }\end{array}$ \\
\hline & $2.529711 E+00$ & 406 & $7.006117 E+02$ & 407 & $1.349032 \mathrm{E}+00$ \\
\hline$z$ & $\begin{array}{l}\text { vp } \mathrm{mf}(\mathrm{kg} / \mathrm{s}) \\
2.292073 \mathrm{E}+00 \\
\text { vp } \mathrm{mf}(\mathrm{kg} / \mathrm{s})\end{array}$ & 416 & $\begin{array}{l}\text { z } 1 \mathrm{q} \mathrm{mf}(\mathrm{kg} / \mathrm{s}) \\
7.008596 \mathrm{E}+02 \\
\text { z } 1 \mathrm{dq} \mathrm{mf}(\mathrm{kg} / \mathrm{s})\end{array}$ & 417 & $\begin{array}{l}z \text { gs vel }(\mathrm{m} / \mathrm{s}) \\
2.535370 \mathrm{E}+00 \\
z \text { gs vel }(\mathrm{m} / \mathrm{s})\end{array}$ \\
\hline
\end{tabular}
id sig.var.
$403 \quad 4.564915 \mathrm{E}+02$
liq temp $(\mathrm{k})$
$408 \quad 1.210095 \mathrm{E}+00$
z lq vel $(\mathrm{m} / \mathrm{s})$
$2.378284 E+00$
$z$ lq vel $(\mathrm{m} / \mathrm{s})$

id sig.var.

$404 \quad 3.328622 \mathrm{E}-01$ void fraction

$4097.031514 \mathrm{E}+02$

z m mfw (kg/s)

$419 \begin{array}{r}7.031517 \mathrm{E}+02 \\ \mathrm{z} \mathrm{m} \mathrm{mfw}(\mathrm{kg} / \mathrm{s})\end{array}$

the component type is a pipe, component number is 4 , first junction number is 4 , and second junction number is 8

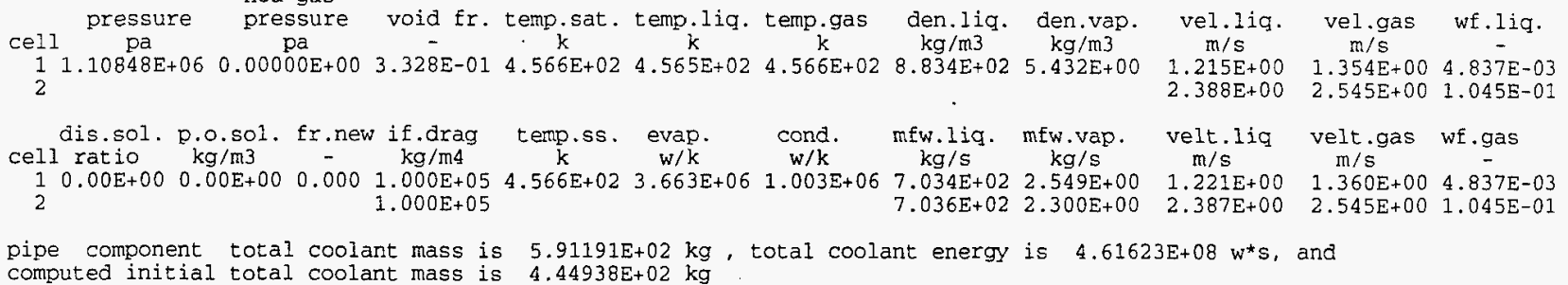

pipe component total coolant mass is $5.91191 \mathrm{E}+02 \mathrm{~kg}$, total coolant energy is $4.61623 \mathrm{E}+08 \mathrm{w} * \mathrm{~s}$, and computed initial total coolant mass is $4.44938 \mathrm{E}+02 \mathrm{~kg}$ 


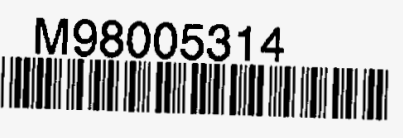

Report Number (14) LA-UR - $98-99$

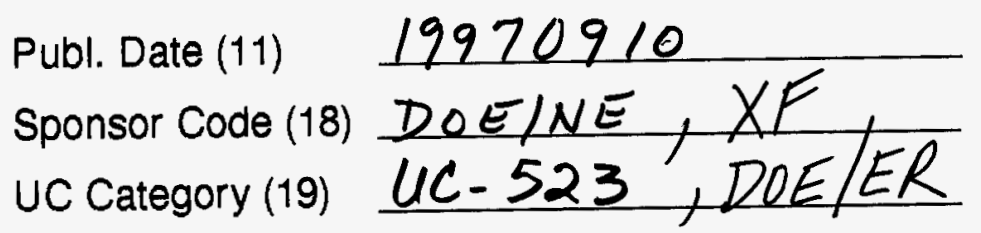

